\title{
13. FLUID INCLUSION STUDIES AS A GUIDE TO THE TEMPERATURE REGIME WITHIN THE TAG HYDROTHERMAL MOUND, $2^{\circ}$ N, MID-ATLANTIC RIDGE ${ }^{1}$
}

\author{
Sven Petersen, ${ }^{2}$ Peter M. Herzig, ${ }^{2}$ and Mark D. Hannington ${ }^{3}$
}

\begin{abstract}
The active Trans-Atlantic Geotraverse (TAG) hydrothermal mound is a mature submarine massive sulfide deposit at the slow-spreading Mid-Atlantic Ridge at $26^{\circ} \mathrm{N}$. Fluid inclusion measurements were conducted on quartz and anhydrite from six boreholes drilled in different areas of the mound to characterize the fluids responsible for the deposition of sulfide-silica breccias and anhydrite and to investigate the vertical and horizontal temperature zonation within an actively forming hydrothermal system. Fluid inclusions in both host minerals are generally two phase liquid/vapor inclusions that homogenize into the liquid phase. Trapping temperatures for quartz and anhydrite from the TAG mound range from $212^{\circ}$ to $390^{\circ} \mathrm{C}$. Salinities vary from 1.9 to $6.2 \mathrm{wt} \% \mathrm{NaCl}$ equivalent for anhydrite and from 4.0 to $6.0 \mathrm{wt} \% \mathrm{NaCl}$ equivalent for quartz. This salinity variation is probably best explained by supercritical phase separation at temperatures above $450{ }^{\circ} \mathrm{C}$ with subsequent remixing of the liquid and the vapor phase during ascent. A zone of anhydrite-rich precipitates recovered at 20 to $35 \mathrm{mbsf}$ below the central Black Smoker Complex (TAG-1) is characterized by trapping temperatures averaging $348^{\circ} \mathrm{C}$ for anhydrite and $358^{\circ} \mathrm{C}$ for quartz, which is slightly below the exit temperature of hydrothermal fluids presently venting at the Black Smoker Complex $\left(360^{\circ}-366^{\circ} \mathrm{C}\right)$. Breccias in the stockwork zone underlying the anhydrite zone were formed at slightly higher temperatures ranging from $327^{\circ}-$ $381^{\circ} \mathrm{C}$ for quartz and from $349^{\circ}$ to $384^{\circ} \mathrm{C}$ for anhydrite. Trapping temperatures vary strongly between different areas of the mound. Fluid inclusions in quartz and anhydrite from the central Black Smoker Complex are characterized by a narrow range of trapping temperatures, whereas other areas drilled on the mound were influenced by lower temperature hydrothermal fluids percolating through the mound or by local entrainment of seawater into the mound. White smokers venting on the southeastern side of the TAG mound are characterized by exit temperatures of $270^{\circ}-300^{\circ} \mathrm{C}$, (Kremlin area, TAG-2). Fluid inclusion measurements in quartz and anhydrite from this area give trapping temperatures in the range of $266^{\circ}-375^{\circ} \mathrm{C}$ with a distinct peak around $340^{\circ} \mathrm{C}$, only somewhat lower than results for the Black Smoker Complex. Trapping temperatures in anhydrite-hosted fluid inclusions in this area show a strong vertical temperature increase. The west side of the mound (TAG-4) is characterized by trapping temperatures ranging from $212^{\circ}$ to $390^{\circ} \mathrm{C}$ showing evidence for seawater entrainment or overprinting by lower temperature hydrothermal events at the sulfide/basalt interface. Samples from the northern side of the mound (TAG-5) exhibit trapping temperatures in the range from $258^{\circ}-383^{\circ} \mathrm{C}$ with a strong vertical temperature increase, indicating additional hightemperature upflow at the northern margin of the mound outside the central Black Smoker Complex.
\end{abstract}

\section{INTRODUCTION}

Processes and characteristics related to the formation of massive sulfide deposits including chimney growth, metal distribution, and fluid chemistry have been studied for several years. A recent summary of these findings is given in Rona and Scott (1993), Hannington et al. (1994) and Herzig and Hannington (1995). All these studies focused on samples taken by submersible, remotely operated vehicle, or dredge from the surface of the deposits. Although in cases such as Galapagos Rift and the Lau Basin, stockwork zones are accessible because of faulting (Embley et al., 1988; Fouquet et al., 1991), the interior of these deposits was, until recently, only known from their fossil analogs in ophiolites. Ocean Drilling Program (ODP) researchers previously drilled the sedimented Middle Valley hydrothermal deposit at the Juan de Fuca Ridge during Leg 139 in 1993, where >94 $\mathrm{m}$ of massive sulfide was recovered in a single hole (Davis, Mottl, Fisher, et al., 1992). In addition, a few shallow cores were recovered at the Snakepit hydrothermal field at the Mid-Atlantic Ridge during Leg 106 in 1985 (Leg 106 Shipboard Scientific Party, 1986). In 1994, during ODP Leg 158, the Trans-Atlantic Geotraverse (TAG) mound at the Mid-Atlantic Ridge $26^{\circ} \mathrm{N}$ was drilled, the first time that deep drilling into an active sediment-free mid-ocean ridge hydrothermal

Herzig, P.M., Humphris, S.E., Miller, D.J., and Zierenberg, R.A. (Eds.), 1998. Proc. ODP, Sci. Results, 158: College Station, TX (Ocean Drilling Program).

${ }^{2}$ Lehrstuhl für Lagerstättenlehre, Institut für Mineralogie, Technische Universität Bergakademie Freiberg, Brennhausgasse 14, D-09596 Freiberg, Federal Republic of Germany. Petersen: petersen@mineral.tu-freiberg.de

${ }^{3}$ Geological Survey of Canada, 601 Booth Street, Ottawa, Ontario K1A 0E8, Canada. system had been accomplished. The TAG hydrothermal field is located on the eastern wall of the rift valley and consists of the active TAG sulfide mound, two relict sulfide zones (Alvin and Mir Zones), and a Mn-oxide-rich low-temperature zone (Rona et al., 1986, 1993; Thompson et al., 1988; Fig. 1). The hydrothermal field covers an area of at least $5 \times 5 \mathrm{~km}$ in water depths ranging from $2300 \mathrm{~m}$ in the lowtemperature Mn-oxide zone to $3700 \mathrm{~m}$ at the active TAG mound. During Leg 158, 17 holes were drilled at 5 different areas of the active TAG mound (Fig. 2) to a maximum depth of $125 \mathrm{mbsf}$ and recovered an assemblage of sulfide \pm anhydrite \pm silica breccias that provides evidence for a long and complex hydrothermal history of the TAG mound (Humphris et al., 1995).

The geochemistry and physical properties of the hydrothermal fluids presently venting at the TAG mound are well known (Campbell et al., 1988; Edmond et al., 1995), although their evolution and spatial distribution are less well understood. Fluid inclusion measurements can give information on the physico-chemical conditions of hydrothermal fluids circulating through the mound before the recent hydrothermal event. The aim of this paper is to describe the petrography and results of microthermometric measurements of fluid inclusions hosted in quartz and anhydrite from a suite of breccias recovered during Leg 158.

\section{GEOLOGICAL SETTING OF THE TAG MOUND}

The active TAG mound is situated near the juncture of the valley floor of the Mid-Atlantic Ridge with the eastern wall at $26^{\circ} 08.23^{\prime} \mathrm{N}$ 
Figure 1. Simplified SeaBeam bathymetry of the MidAtlantic ridge at $26^{\circ} \mathrm{N}$, showing the location of volcanic domes, the active TAG mound, the two inactive sulfide deposits (Mir and Alvin zones), and the low-temperature (LT) Mn-oxide zone within the TAG hydrothermal field (from Rona et al., 1993).
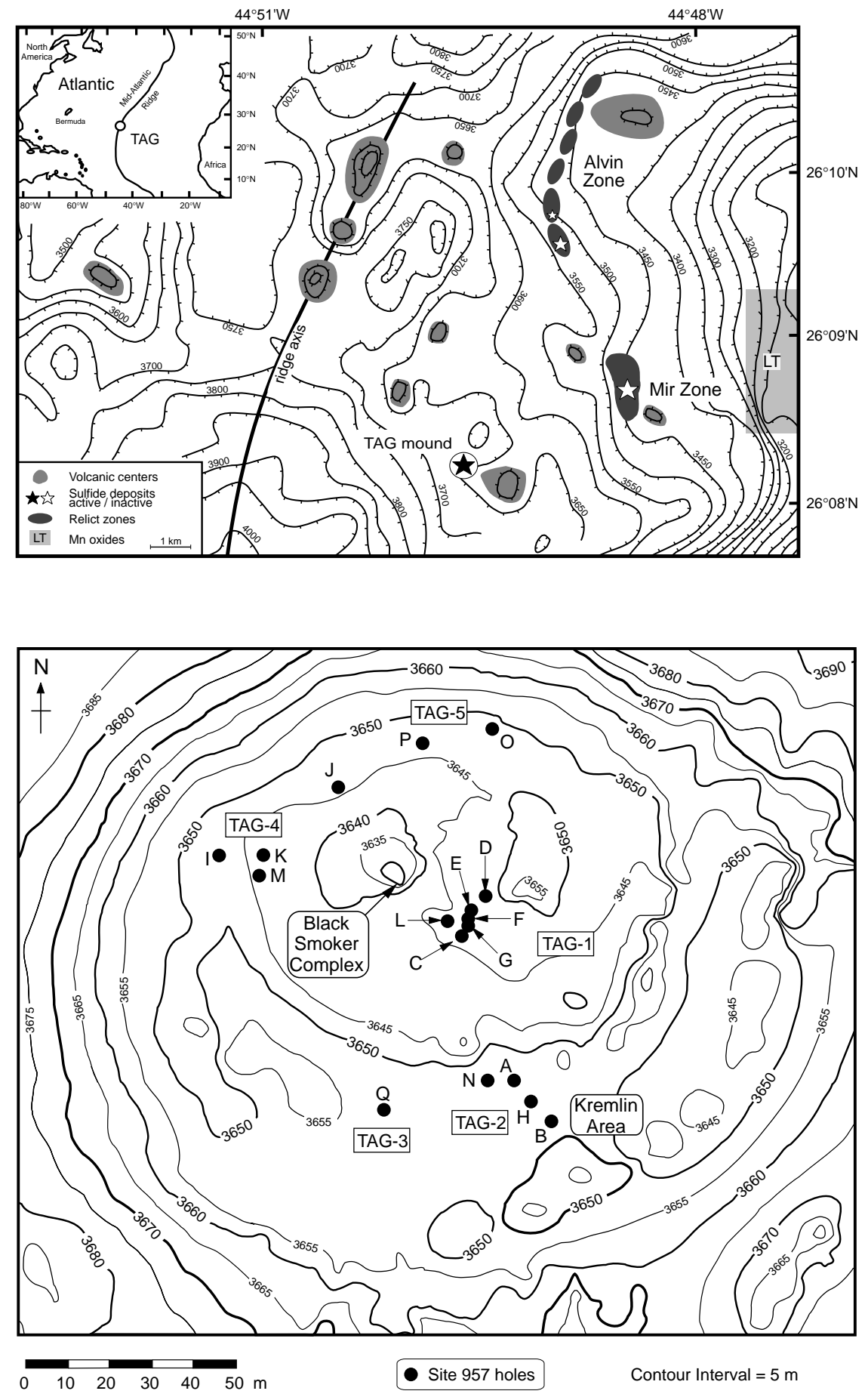

Figure 2. Detailed bathymetric contour map of the active TAG mound, showing the central Black Smoker Complex, the area of white smoker venting (Kremlin area) and the location of holes drilled during ODP Leg 158 (modified from Humphris et al., 1995).
Complex, TAG-1) $10 \mathrm{~m}$ high, narrowing from $20 \mathrm{~m}$ in diameter at its base to $5-10 \mathrm{~m}$ in diameter at the summit, is situated just to the west of the center of the upper platform. The top of this structure is the focus of intense hydrothermal black smoker discharge at temperatures of up to $360^{\circ}-366^{\circ} \mathrm{C}$ (Edmond et al., 1995). Recent photometric surveys showed an elongated north-northwest-trending depression, up to $10 \mathrm{~m}$ deep and measuring $25 \times 20 \mathrm{~m}$, east of the Black Smoker Complex that had not been observed in earlier dives. This depression is probably related to a collapse feature and anhydrite is exposed along its walls (Kleinrock et al., 1996). Black smoker activity occurs at the margin of the depression, along small rift-parallel fissures, and is not restricted to the Black Smoker Complex. Age-dating of massive sulfides indicates that high-temperature hydrothermal activity at the active TAG mound began approximately $50 \mathrm{ka}$ with hydrother- 
mal episodes occurring every 5000-6000 yr (Lalou et al., 1990, 1995). The present hydrothermal activity may have initiated only 50 yr ago (Lalou et al., 1993).

\section{METHODS}

The microthermometric studies were conducted on doubly polished thick sections and single grains using a USGS heating/freezing stage and a Linkam THMS 600 stage (Shepherd, 1981). Both stages were calibrated using synthetic standards with known melting and homogenization temperatures $\left(-56.2^{\circ}, 0.0^{\circ}, 374.1^{\circ} \mathrm{C}\right)$. Homogenization and dissolution temperatures were obtained from replicate heating and freezing measurements. Samples were progressively heated to avoid decrepitation or alteration of the inclusions from stretching and leaking. Results reported for anhydrite are single measurements performed on inclusions within clusters of inclusions that display similar phase ratios and homogenization temperatures. Measured fluid inclusions in quartz, however, are often isolated and, although several grains per sample have been measured, might not be representative for the sample. The accuracy of the measurements is estimated to $\pm 2^{\circ} \mathrm{C}$ at $350^{\circ} \mathrm{C}$ and $\pm 0.2^{\circ} \mathrm{C}$ below $0^{\circ} \mathrm{C}$. Salinities were obtained using the equation given by Bodnar (1993). Fluid inclusions were cooled until they were completely frozen and then heated at steps of $20^{\circ}, 5^{\circ}$, and $1^{\circ} \mathrm{C}$ per min. Homogenization temperatures were pressure corrected using the equation of Zhang and Frantz (1987).

\section{SAMPLE DESCRIPTION}

A detailed description of the different lithologies found during Leg 158 is provided by Humphris et al. (1995), and only a short summary of sample types is given here. The upper $15 \mathrm{~m}$ of the TAG mound consists of massive pyrite and pyrite breccias, which are partly overlain by Fe-oxyhydroxides and cherts. Between $20 \mathrm{mbsf}$ and 35 mbsf, an anhydrite-rich zone was encountered to the east and the north of the central Black Smoker Complex. Nodular siliceous pyrite-anhydrite breccias occur mainly between 19.5 and 23 mbsf and are characterized by numerous rounded massive pyrite and siliceous clasts in a matrix of anhydrite and minor quartz (Pl. 1, Fig. 1). The rounded siliceous clasts contain disseminated pyrite and pyrite aggregates in a matrix of dark-gray quartz that are similar to pyrite-silica breccias further downcore. Anhydrite veins are abundant and reach up to $40 \mathrm{~cm}$ in thickness. A 10-m-thick transition between the anhydrite zone and underlying pyrite-silica breccias occurs between 35 and 45 mbsf. In this transition, silica becomes the dominant matrix component and the amount of anhydrite decreases. Pyrite-silica breccias mark the beginning of the stockwork zone. They are generally matrix-supported and contain abundant altered wallrock fragments. Single wallrock fragments have also been observed higher up in the core (Alt and Teagle, Chap. 21, this volume). Pyrite-silica breccias in the TAG-2 area (see Fig. 2) are characterized by large subrounded siliceous clasts containing variable amounts of disseminated pyrite and sometimes Fe-oxide (Pl. 1, Fig. 2). These clasts occur in a very finegrained matrix of quartz with disseminated pyrite and minor chalcopyrite. Pyrite-silica breccias in the TAG-4 area often contain medium-gray, siliceous fragments and smaller quartz-pyrite fragments in a matrix of dark-gray quartz, colored by fine-grained disseminated sulfides (Pl. 1, Fig. 3). Remnants of nodular pyrite are present. The siliceous fragments show diffuse or sharp contacts with the matrix and resemble silicified wallrock fragments further downcore, but their igneous texture has often been destroyed. No anhydrite has been found in the TAG-4 area. Pyrite-silica breccias grade into silicified wallrock breccias and sericitized and/or chloritized basalt breccias at depth. In contrast to pyrite-silica breccias, silicified wallrock breccias are clast-supported and are characterized by buff-colored, strongly silicified wallrock fragments that contain relict igneous textures. Some wallrock fragments are clearly recognizable; others are almost com- pletely silicified (Pl. 1, Fig. 4). Anhydrite veins are abundant in most samples from the stockwork zone and commonly rim samples. Pyritization and the formation of chalcopyrite are commonly associated with these veins. Sericitized and chloritized basalt breccias further downcore consist of wallrock fragments that have been intensely altered to paragonite or chlorite (Honnorez et al., Chap. 18, this volume) with minor quartz. The fragments are often intensely fractured and crosscut by quartz-pyrite veins.

\section{RESULTS Petrography}

Fluid inclusions in quartz and anhydrite from different lithologies including pyrite-anhydrite breccias, nodular siliceous pyrite-anhydrite breccias, pyrite-silica \pm anhydrite breccias, silicified wallrock breccias, and sericitized basalt breccias from different areas of the TAG mound have been investigated. Quartz in samples from the TAG mound is generally fine to medium-grained $(<50-200 \mu \mathrm{m})$ and milky, but cryptocrystalline quartz and chalcedony occur. Quartz in pyrite-silica breccias often shows multiple generations of micrometer-scale banding that might be explained by hydrothermal recrystallization of a banded amorphous silica precursor. Fluid inclusions in quartz with growth banding are extremely rare and small $(<1 \mu \mathrm{m})$. This banded quartz is overgrown by coarse-grained $(200-400 \mu \mathrm{m})$ transparent quartz without banding that also occurs as infilling of vugs. This quartz is generally the latest quartz generation in samples from TAG. Euhedral quartz has also been observed as small isolated grains $(20-100 \mu \mathrm{m})$ in a cryptocrystalline matrix in breccias from the TAG-4 area. These quartz grains are characterized by a strong zonation on a micrometer-scale and rarely contain measurable fluid inclusions (Pl. 2, Fig. 2). The chalcedonic matrix often shows micrometerscale banding similar to that observed in samples from other areas of the mound. Anhydrite at the TAG mound is generally medium- to coarse-grained (up to $3 \mathrm{~mm}$ ) and, late in the paragenesis, often filling void space or veins in surrounding breccias. Anhydrite is either transparent or slightly colored by abundant extremely small inclusions $(<<1 \mu \mathrm{m})$. Some anhydrite grains especially in samples collected near the surface of TAG-1 and TAG-2, are mainly fine- to mediumgrained and show dissolution along grain boundaries caused by the retrograde solubility of anhydrite in seawater (Blount and Dickson, 1969).

The classification of Roedder (1984) was used to discriminate primary and secondary fluid inclusions. Fluid inclusions in both host minerals are generally two-phase liquid/vapor inclusions that homogenize into the liquid phase. The occurrence of few secondary vaporrich and/or liquid-rich or even single-phase liquid or vapor fluid inclusions in close proximity is interpreted as the result of necking of inclusions rather then trapping of boiling fluids. Primary fluid inclusions in quartz are generally elliptical to circular and generally $<10$ $\mu \mathrm{m}$ with only a few larger inclusions. Small inclusions sometimes appear as groups in the core of euhedral quartz, whereas large inclusions are isolated and can occupy a substantial area of a quartz grain (Pl. 2, Fig. 1). Angular fluid inclusions have been observed interstitial to quartz grains in all areas of the TAG mound (Pl. 2, Fig. 2). Interstitial fluid inclusions range from $<10$ to $50 \mu \mathrm{m}$ in size, but are typically between 15 and $30 \mu \mathrm{m}$ in diameter. Most salinity measurements in quartz were conducted on these interstitial fluid inclusions and reproducibility of the results is very good. In rare cases, opaque minerals have been found in these inclusions. Secondary inclusions in quartz are irregular or oval in shape, often thin and generally occur as planar arrays, but a few large isolated inclusions have been observed.

Primary fluid inclusions in anhydrite occur parallel to crystal faces and are either subrounded, square, rectangular or needle-shaped, ranging in size from 1 to $40 \mu \mathrm{m}$ (Pl. 2, Fig. 3). Most primary inclusions range from 1 to $20 \mu \mathrm{m}$. Secondary inclusions occur as very small square, angular, subangular or irregular inclusions along healed 
microfractures. Most secondary inclusions are $<<1 \mu \mathrm{m}$ in size and not measurable. Some of these inclusions show extreme variability in homogenization temperatures. Anhydrite crystals are often fractured, broken, and healed by transparent anhydrite (see Pl. 2, Fig. 4). Fluid inclusion trails are often truncated by the fractures, but some reach into the newly formed anhydrite.

\section{Microthermometry}

Homogenization temperatures (Th) for all inclusions in quartz and anhydrite from the TAG mound range from $191^{\circ}$ to $364^{\circ} \mathrm{C}$ (avg $=326^{\circ} \mathrm{C}, \mathrm{SD}=29.8, \mathrm{n}=574$; Fig. $3 \mathrm{~A}$ ). Corresponding final ice melting $(\mathrm{Tm})$ occurred at temperatures between $-1.1^{\circ}$ and $-3.8^{\circ} \mathrm{C}($ avg $=$ $-2.7^{\circ} \mathrm{C}, \mathrm{SD}=0.6, \mathrm{n}=125$; Fig. $3 \mathrm{~B}$ ). A summary of the microthermometric results is given in Table 1. Homogenization temperatures have been corrected for pressure effects using the equation of Zhang and Frantz (1987) for the system $\mathrm{NaCl}-\mathrm{H}_{2} \mathrm{O}$ assuming hydrostatic pressure. Pressure correction results in trapping temperatures (Tt) from $212^{\circ}$ to $390^{\circ} \mathrm{C}$ for all inclusions from the TAG mound.

Salinities were calculated from freezing-point depression using the equation:

$$
\text { Salinity }=0.00+1.78 \theta-0.0442 \theta^{2}+0.000557 \theta^{3}
$$

for the $\mathrm{H}_{2} \mathrm{O}-\mathrm{NaCl}$ system (Bodnar, 1993), where $\theta$ is the freezingpoint depression.

Calculated salinities range from 1.9 to $6.2 \mathrm{wt} \% \mathrm{NaCl}$ equivalent (avg $=4.5 \mathrm{wt} \% \mathrm{NaCl}$ equivalent, $\mathrm{SD}=0.9, \mathrm{n}=125)$ which equals 0.6 to 2 times seawater salinity ( $3.2 \mathrm{wt} \% \mathrm{NaCl}$ equivalent; Fig. 3C). Quartz is solely characterized by salinities above seawater salinity, ranging from 4.0 to $6.0 \mathrm{wt} \% \mathrm{NaCl}$ equivalent (avg $=5.0 \mathrm{wt} \% \mathrm{NaCl}$ equivalent, $\mathrm{SD}=0.6, \mathrm{n}=43$ ). Systematic salinity variations between primary and secondary fluid inclusions have not been observed.

Although the microthermometric results show generally high trapping temperatures for all samples from the TAG mound, differences between the individual areas occur. The TAG- 1 area is situated next to the central active high temperature Black Smoker Complex (BSC) close to the proposed upflow zone, and is characterized by a narrow range of homogenization temperatures in quartz and anhydrite, ranging from $301^{\circ}$ to $358^{\circ} \mathrm{C}\left(\operatorname{avg}=334^{\circ} \mathrm{C}, \mathrm{SD}=13.3, \mathrm{n}=333\right.$, Fig. 4). A systematic variation in homogenization temperatures between secondary and primary inclusions or between different inclusion types within anhydrite or quartz has not been observed at TAG1. Samples from the anhydrite-rich zone (20-35 mbsf) in the center of the mound show homogenization temperatures in the range from $306^{\circ}$ to $350^{\circ} \mathrm{C}$ for anhydrite $\left(\operatorname{avg}=322^{\circ} \mathrm{C}, \mathrm{SD}=10.4, \mathrm{n}=64\right)$ and from $311^{\circ}$ to $342^{\circ} \mathrm{C}$ for quartz $\left(\operatorname{avg}=332^{\circ} \mathrm{C}, \mathrm{SD}=7.4, \mathrm{n}=32\right.$ ). Fluid inclusions in quartz and anhydrite from the subseafloor stockwork zone (below $35 \mathrm{mbsf}$ ) show a moderate increase in homogenization temperatures, ranging from $323^{\circ}$ to $358^{\circ} \mathrm{C}$ for anhydrite (avg = $347^{\circ} \mathrm{C}, \mathrm{SD}=5.9, \mathrm{n}=63$ ) and from $301^{\circ}$ to $355^{\circ} \mathrm{C}$ for quartz (avg = $\left.344^{\circ} \mathrm{C}, \mathrm{SD}=10.2, \mathrm{n}=174\right)$. This vertical temperature zonation becomes evident when trapping temperatures are plotted vs. depth (Fig. 5). Trapping temperatures in the anhydrite zone are lower for anhydrite then for quartz, whereas at depth the trapping temperatures for both host minerals are almost the same or even higher for anhydrite.

Samples from the southeastern side of the mound (Kremlin area, TAG-2) show homogenization temperatures in the range from $242^{\circ}$ to $349^{\circ} \mathrm{C}\left(\mathrm{avg}=314^{\circ} \mathrm{C}, \mathrm{SD}=24.3, \mathrm{n}=103\right.$; Fig. $\left.6 \mathrm{~A}\right)$. In contrast to the narrow profile of the TAG-1 data, the frequency distribution of the TAG-2 results shows a wider range and the main peak is shifted to lower temperatures. Homogenization temperatures vary strongly with depth. Fluid inclusions in anhydrite from a pyrite-anhydrite breccia collected near the surface (Sample 158-957H-1N-1, 42-47 $\mathrm{cm})$ show homogenization temperatures of $242^{\circ}-295^{\circ} \mathrm{C}($ avg $=$ $265^{\circ} \mathrm{C}, \mathrm{n}=9$ ). Quartz and anhydrite from a pyrite-silica breccia and
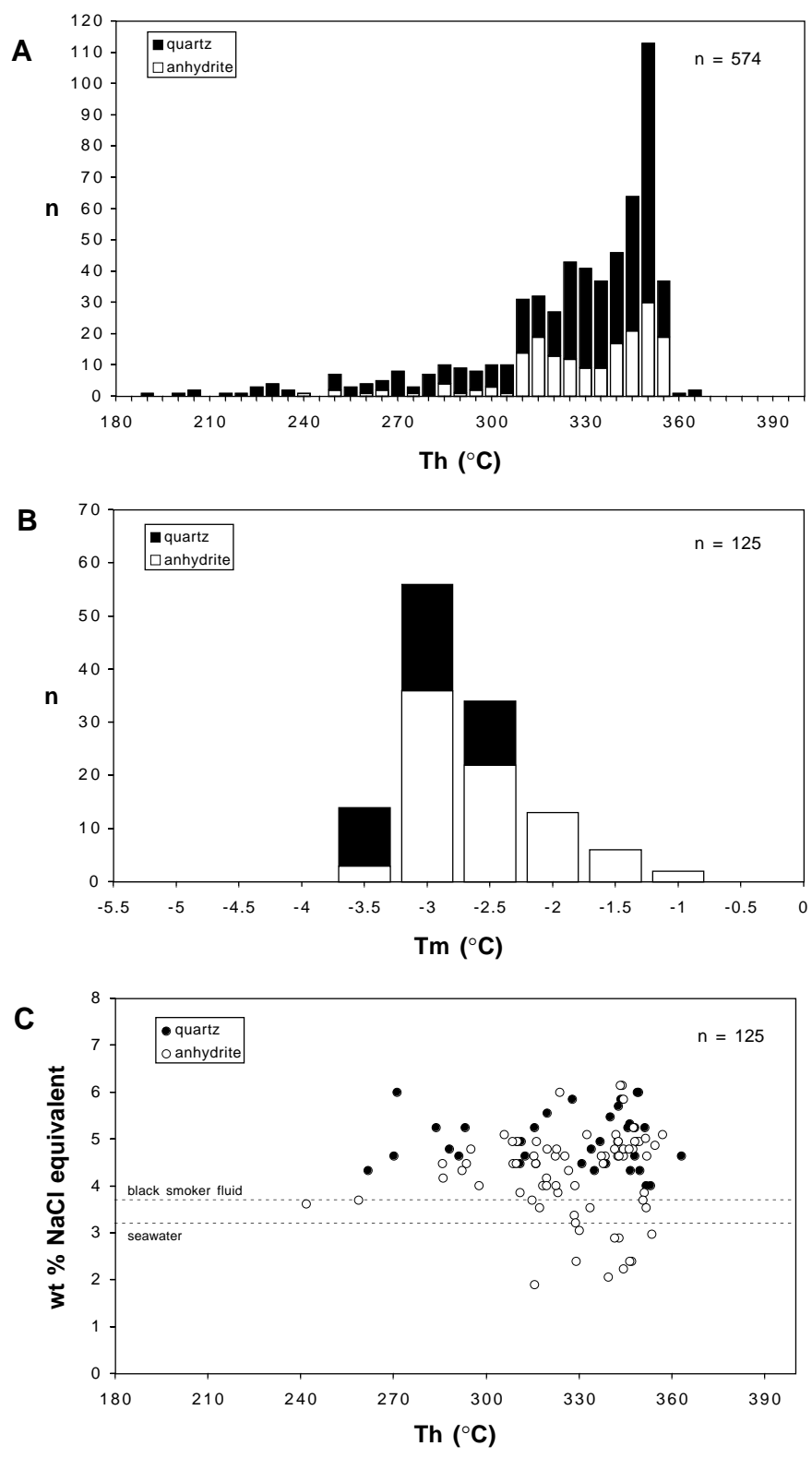

Figure 3. Microthermometric results for fluid inclusions in quartz and anhydrite from the TAG mound. A. Frequency histogram of homogenization temperatures (Th). B. Frequency histogram of final ice melting (Tm). C. Homogenization temperatures and corresponding salinities. $\mathrm{n}=$ number of measurements.

a silicified wallrock breccia at $28 \mathrm{mbsf}$ give homogenization temperatures in the range from $262^{\circ}$ to $349^{\circ} \mathrm{C}$ with a distinct peak around $320^{\circ} \mathrm{C}$. Primary fluid inclusions in quartz from both samples show similar homogenization temperatures ranging from $302^{\circ}$ to $339^{\circ} \mathrm{C}$ (avg $=324^{\circ} \mathrm{C}, \mathrm{n}=27$, Sample $\left.158-957 \mathrm{H}-5 \mathrm{~N}-1,73-75 \mathrm{~cm}\right)$ and $303^{\circ}$ to $349^{\circ} \mathrm{C}\left(\right.$ avg $=326^{\circ} \mathrm{C}, \mathrm{n}=28$, Sample $\left.158-957 \mathrm{H}-5 \mathrm{~N}-2,75-80 \mathrm{~cm}\right)$ respectively. Homogenization temperatures in secondary fluid inclusions in quartz are generally lower than in primary inclusions and range from $262^{\circ}$ to $308^{\circ} \mathrm{C}$ with averages of $284^{\circ} \mathrm{C}(\mathrm{n}=13)$ and $290^{\circ} \mathrm{C}$ $(n=4)$ in those samples. Vein-related anhydrite is characterized by average homogenization temperatures of $319^{\circ} \mathrm{C}$ (range from $311^{\circ}$ $335^{\circ} \mathrm{C}, \mathrm{n}=13$ ), which is comparable to the results of the primary fluid inclusions in quartz. Anhydrite from a pyrite-silica breccia collect- 
Table 1. Description and microthermometric results of fluid inclusions in silica-rich breccias and anhydrite from the TAG hydrothermal mound.

\begin{tabular}{|c|c|c|c|c|c|c|c|c|c|c|c|c|c|}
\hline \multirow{2}{*}{$\begin{array}{l}\text { Core, section, } \\
\text { interval }(\mathrm{cm})\end{array}$} & \multirow{2}{*}{$\begin{array}{l}\text { Depth } \\
\text { (mbsf) }\end{array}$} & \multirow[b]{2}{*}{ Sample type, description } & \multirow[b]{2}{*}{ Host } & \multirow[b]{2}{*}{ Comments } & \multirow[b]{2}{*}{$\mathrm{n}$} & \multicolumn{3}{|c|}{$\operatorname{Th}\left({ }^{\circ} \mathrm{C}\right)$} & \multirow[b]{2}{*}{$\mathrm{n}$} & \multicolumn{3}{|c|}{$\operatorname{Tm}\left({ }^{\circ} \mathrm{C}\right)$} & \multirow{2}{*}{$\begin{array}{l}\mathrm{NaCl} \\
\mathrm{wt} \%\end{array}$} \\
\hline & & & & & & Range & Average & SD & & Range & Average & SD & \\
\hline \multicolumn{14}{|l|}{ TAG-1 } \\
\hline \multirow[t]{2}{*}{$7 \mathrm{~N}-2,13-16$} & \multirow[t]{2}{*}{20.83} & \multirow{2}{*}{$\begin{array}{l}\text { Nodular siliceous pyrite-anhydrite breccia; rounded } \\
\text { pyrite and pyrite-quartz fragments in anhydrite matrix } \\
\text { with disseminated very fine-grained pyrite. }\end{array}$} & qtz & $\begin{array}{l}\text { Fluid inclusions in fine-grained subhedral quartz from } \\
\text { rounded pyrite-quartz aggregates. }\end{array}$ & 10 & $328-342$ & 331 & 3.9 & 1 & - & -2.7 & - & 4.5 \\
\hline & & & anh & $\begin{array}{l}\text { Primary and secondary fluid inclusions in coarse- } \\
\text { grained matrix anhydrite }\end{array}$ & 25 & $308-326$ & 318 & 5.9 & 4 & $-1.1-3.7$ & -2.5 & 1.1 & 4.2 \\
\hline $7 \mathrm{~N}-2,107-110$ & 21.81 & $\begin{array}{l}\text { Anhydrite vein; contact between anhydrite veins and } \\
\text { nodular pyrite-silica breccia with anhydrite cementing } \\
\text { nodular pyrite aggregates. }\end{array}$ & anh & Primary angular inclusions in matrix anhydrite. & 4 & $315-327$ & 321 & 5.7 & 7 & $-2.2-2.8$ & -2.5 & 0.3 & 4.2 \\
\hline $7 \mathrm{~N}-2,148-149$ & 22.12 & $\begin{array}{l}\text { Nodular siliceous pyrite-anhydrite breccia; rounded } \\
\text { pyrite aggregates in banded anhydrite matrix dusted by } \\
\text { very fine-grained disseminated pyrite. }\end{array}$ & anh & $\begin{array}{l}\text { Primary and secondary fluid inclusions in medium- } \\
\text { grained anhydrite overgrowing pyrite aggregates. }\end{array}$ & 16 & $306-338$ & 316 & 9.1 & 13 & $-2.7-3.1$ & -2.9 & 0.1 & 4.8 \\
\hline $11 \mathrm{~N}-1,70-74$ & 31.39 & $\begin{array}{l}\text { Pyrite-silica-anhydrite breccia; rounded pyrite aggregates } \\
\text { in siliceous matrix with thin pyritization halo and late, } \\
\text { vein-related anhydrite. }\end{array}$ & qtz & $\begin{array}{l}\text { Fluid inclusions at grain boundaries and secondary fluid } \\
\text { inclusions in siliceous matrix. }\end{array}$ & 10 & $311-341$ & 329 & 11.2 & - & - & - & - & - \\
\hline \multirow[t]{2}{*}{$11 \mathrm{~N}-2,4-6$} & \multirow[t]{2}{*}{32.19} & \multirow{2}{*}{$\begin{array}{l}\text { Pyrite-silica-anhydrite breccia; vein-related sulfidation } \\
\text { rim overgrowing pyrite-quartz intergrowth. Vein-related } \\
\text { anhydrite is cementing fragments of the sulfidation rim } \\
\text { and the early aggregates. }\end{array}$} & $\mathrm{qtz}$ & $\begin{array}{l}\text { Fluid inclusions at grain boundaries and primary } \\
\text { inclusions in fine-grained }(100-200 \mu \mathrm{m}) \text { quartz. }\end{array}$ & 12 & $329-341$ & 336 & 3.5 & 1 & - & -3.0 & - & 5.0 \\
\hline & & & anh & $\begin{array}{l}\text { Primary fluid inclusions in coarse-grained late anhydrite } \\
\text { vein. }\end{array}$ & 19 & $314-350$ & 332 & 10.0 & 12 & $-1.4-3.1$ & -2.4 & 0.6 & 4.1 \\
\hline \multirow[t]{2}{*}{$12 \mathrm{~N}-2,81-83$} & \multirow[t]{2}{*}{36.23} & \multirow{2}{*}{$\begin{array}{l}\text { Pyrite-silica-anhydrite breccia: light gray quartz-pyrite } \\
\text { breccia with minor network of late anhydrite crosscutting } \\
\text { the breccia. }\end{array}$} & qtz & $\begin{array}{l}\text { Primary fluid inclusions in fine-grained }(20-50 \mu \mathrm{m}) \\
\text { subhedral quartz. }\end{array}$ & 16 & $337-351$ & 346 & 3.5 & 4 & $-3.2-3.4$ & -3.3 & 0.1 & 5.4 \\
\hline & & & anh & $\begin{array}{l}\text { Primary fluid inclusions in coarse-grained late } \\
\text { anhydrite. }\end{array}$ & 11 & $335-352$ & 344 & 4.3 & 5 & $-2.8-3.8$ & -3.4 & 0.5 & 5.5 \\
\hline $13 \mathrm{~N}-1,130-132$ & 38.47 & $\begin{array}{l}\text { Pyrite-silica breccia; medium gray quartz matrix } \\
\text { surrounding large light gray siliceous fragments. Sample } \\
\text { is rimmed by vein-related pyrite-anhydrite. }\end{array}$ & qtz & $\begin{array}{l}\text { Fluid inclusions at grain boundaries and primary } \\
\text { rounded inclusions in late, transparent, medium-grained } \\
(50-150 \mu \mathrm{m}) \text { quartz filling vugs. }\end{array}$ & 10 & $347-350$ & 349 & 1.3 & - & - & - & - & - \\
\hline \multirow[t]{2}{*}{$14 \mathrm{~N}-2,13-16$} & \multirow[t]{2}{*}{40.90} & \multirow[t]{2}{*}{$\begin{array}{l}\text { Silicified wallrock breccia: silicified wallrock fragments } \\
\text { in a matrix of white quartz and vein-related anhydrite. } \\
\text { Strong pyritization halo with some chalcopyrite }\end{array}$} & qtz & $\begin{array}{l}\text { Primary and } \\
\text { secondary fluid inclusions in large }(200-400 \mu \mathrm{m}) \\
\text { transparent quartz filling vugs. }\end{array}$ & $\begin{array}{l}3 \\
8\end{array}$ & $\begin{array}{l}324-347 \\
301-339\end{array}$ & $\begin{array}{l}332 \\
317\end{array}$ & $\begin{array}{l}15.2 \\
14.7\end{array}$ & $\overline{-}$ & 二 & 二 & $\overline{-}$ & 二 \\
\hline & & & anh & $\begin{array}{l}\text { Primary fluid inclusions in coarse-grained vein-related } \\
\text { anhydrite. }\end{array}$ & 6 & $342-348$ & 345 & 2.4 & 6 & $-1.3-3.2$ & -1.8 & 0.7 & 3.0 \\
\hline \multirow[t]{2}{*}{$15 \mathrm{~N}-2,18-22$} & \multirow[t]{2}{*}{43.16} & \multirow{2}{*}{$\begin{array}{l}\text { Silicified wallrock breccia; small silicified wallrock } \\
\text { fragments in pyrite-silica matrix. Void around } 1.5-\mathrm{cm} \\
\text { large pyrite nodule is filled with late anhydrite. }\end{array}$} & $\mathrm{qtz}$ & $\begin{array}{l}\text { Primary and secondary inclusions in transparent quartz } \\
\text { cementing wallrock fragments. }\end{array}$ & 12 & $336-355$ & 345 & 4.9 & 3 & $-3.5-3.7$ & -3.6 & 0.1 & 5.9 \\
\hline & & & anh & $\begin{array}{l}\text { Primary and secondary fluid inclusions in coarse- } \\
\text { grained late anhydrite. }\end{array}$ & 29 & $323-358$ & 347 & 7.2 & 13 & $-1.2-3.0$ & -2.4 & 0.5 & 4.1 \\
\hline $15 \mathrm{~N}-3,133-138$ & 45.54 & $\begin{array}{l}\text { Silicified wallrock breccia; silicified wallrock fragments } \\
\text { and nodular pyrite aggregates in a matrix of dark quartz } \\
\text { with late coarser-grained white quartz and some vein- } \\
\text { related anhydrite. }\end{array}$ & qtz & $\begin{array}{l}\text { Fluid inclusions at grain boundaries, primary and } \\
\text { secondary inclusions in late transparent quartz. }\end{array}$ & 6 & $323-350$ & 339 & 10.7 & 1 & - & -2.6 & - & 4.3 \\
\hline $\begin{array}{l}\text { 158-957E- } \\
1 \mathrm{R}-1,5-7\end{array}$ & 31.50 & $\begin{array}{l}\text { Nodular pyrite-silica breccia; pyrite nodules }(<3 \mathrm{~mm}) \text { and } \\
\text { disseminated fine-grained pyrite in a light gray quartz } \\
\text { matrix }\end{array}$ & qtz & $\begin{array}{l}\text { Fluid inclusions at grain boundaries, primary and } \\
\text { secondary inclusions in late transparent quartz. }\end{array}$ & 14 & $346-352$ & 348 & 1.6 & 2 & $-2.4-2.6$ & -2.5 & 0.1 & 4.2 \\
\hline \multirow[t]{2}{*}{$4 \mathrm{R}-1,1-4$} & \multirow[t]{2}{*}{49.00} & \multirow{2}{*}{$\begin{array}{l}\text { Silicified wallrock breccia; small (up to } 5 \mathrm{~mm} \text { ) pyrite } \\
\text { nodules in silicified wallrock material. A millimeter-thin } \\
\text { chalcopyrite halo at the outer surface of the sample is } \\
\text { rimmed by late anhydrite. }\end{array}$} & qtz & $\begin{array}{l}\text { Fluid inclusions at grain boundaries and secondary } \\
\text { inclusions in quartz surrounding pyrite aggregates. }\end{array}$ & 19 & $319-353$ & 344 & 10.1 & 2 & $-2.4-3.7$ & -3.1 & 0.9 & 5.1 \\
\hline & & & anh & $\begin{array}{l}\text { Primary and secondary fluid inclusions in coarse- } \\
\text { grained late anhydrite. }\end{array}$ & 10 & $348-354$ & 352 & 1.9 & - & - & - & - & - \\
\hline $4 \mathrm{R}-1,20-23$ & 49.18 & $\begin{array}{l}\text { Silicified wallrock breccia; pyrite nodules and } \\
\text { disseminated fine-grained pyrite in altered wallrock } \\
\text { material and light gray quartz. }\end{array}$ & qtz & $\begin{array}{l}\text { Primary and secondary fluid inclusions in quartz } \\
\text { surrounding pyrite aggregates. }\end{array}$ & 14 & $345-352$ & 350 & 2.0 & - & - & - & - & - \\
\hline
\end{tabular}




\begin{tabular}{|c|c|c|c|c|c|c|c|c|c|c|c|c|c|}
\hline \multirow{2}{*}{$\begin{array}{l}\text { Core, section, } \\
\text { interval }(\mathrm{cm})\end{array}$} & \multirow{2}{*}{$\begin{array}{l}\text { Depth } \\
\text { (mbsf) }\end{array}$} & \multirow[b]{2}{*}{ Sample type, description } & \multirow[b]{2}{*}{ Host } & \multirow[b]{2}{*}{ Comments } & \multirow[b]{2}{*}{$\mathrm{n}$} & \multicolumn{3}{|c|}{ Th $\left({ }^{\circ} \mathrm{C}\right)$} & \multirow[b]{2}{*}{$\mathrm{n}$} & \multicolumn{3}{|c|}{$\operatorname{Tm}\left({ }^{\circ} \mathrm{C}\right)$} & \multirow{2}{*}{$\begin{array}{l}\mathrm{NaCl} \\
\mathrm{wt} \%\end{array}$} \\
\hline & & & & & & Range & Average & SD & & Range & Average & SD & \\
\hline \multirow[t]{2}{*}{ 7R-1, 14-18 } & \multirow[t]{2}{*}{68.42} & \multirow[t]{2}{*}{$\begin{array}{l}\text { Silicified wallrock breccia; sericitized wallrock } \\
\text { fragments with very fine-grained disseminated pyrite } \\
\text { dusting cut by quartz-pyrite and pyrite veinlets }(\mathrm{mm}) \text {. }\end{array}$} & qtz & $\begin{array}{l}\text { Fluid inclusions at grain boundaries and secondary } \\
\text { inclusions in medium-grained quartz surrounding pyrite } \\
\text { aggregates. }\end{array}$ & 15 & $315-353$ & 341 & 14.4 & 2 & $-3.2-3.2$ & -3.2 & - & 5.3 \\
\hline & & & anh & $\begin{array}{l}\text { Small primary inclusions in single anhydrite grain filling } \\
\text { void space }\end{array}$ & 7 & $347-349$ & 348 & 0.5 & - & - & - & - & - \\
\hline $8 R-1,15-20$ & 72.95 & $\begin{array}{l}\text { Silicified wallrock breccia; subrounded light gray quartz- } \\
\text { pyrite fragments containing Fe-oxyhydroxides and } \\
\text { silicified wallrock fragments in dark siliceous matrix. }\end{array}$ & qtz & $\begin{array}{l}\text { Fluid inclusions at grain boundaries, primary and } \\
\text { secondary inclusions in medium-grained }(50-150 \mu \mathrm{m}) \\
\text { quartz surrounding pyrite aggregates and wallrock } \\
\text { fragments. }\end{array}$ & 11 & $348-353$ & 350 & 2.3 & - & - & - & - & - \\
\hline $12 \mathrm{R}-1,26-28$ & 92.00 & $\begin{array}{l}\text { Silicified wallrock breccia; light gray siliceous fragments } \\
\text { in a dark siliceous matrix. Disseminated pyrite } \\
\text { throughout the sample and as vein-related pyritization } \\
\text { halo. Transparent quartz in vugs. }\end{array}$ & qtz & $\begin{array}{l}\text { Primary and secondary fluid inclusions in medium- } \\
\text { grained }(<100 \mu \mathrm{m}) \text { quartz filling vugs in } \\
\text { cryptocrystalline banded to colloform quartz }\end{array}$ & 9 & $322-354$ & 346 & 9.2 & - & - & - & - & - \\
\hline $15 \mathrm{R}-1,1-4$ & 106.50 & $\begin{array}{l}\text { Sericitized and chloritized basalt breccia: buff-colored } \\
\text { wallrock fragments in a white siliceous matrix with } \\
\text { abundant disseminated pyrite }\end{array}$ & qtz & $\begin{array}{l}\text { Small fluid inclusions at grain boundaries and secondary } \\
\text { inclusions in medium-grained, milky quartz surrounding } \\
\text { pyrite aggregates. }\end{array}$ & 17 & $328-354$ & 349 & 6.41 & - & - & 3.2 & - & 5.3 \\
\hline $17 \mathrm{R}-1,16-18$ & 116.19 & $\begin{array}{l}\text { Sericitized and chloritized basalt breccia; strongly } \\
\text { sericitized basalt breccia cut by two generations of } \\
\text { quartz-pyrite veins. }\end{array}$ & qtz & $\begin{array}{l}\text { Fluid inclusions related to grain boundaries and rounded } \\
\text { primary inclusions in transparent quartz (up to } 100 \mu \mathrm{m} \text { ) } \\
\text { related to two different sets of veins. }\end{array}$ & 20 & $333-355$ & 345 & 6.1 & - & - & - & - & - \\
\hline \multicolumn{14}{|l|}{$\begin{array}{l}\text { TAG-2 } \\
158-957 \mathrm{H}\end{array}$} \\
\hline $1 \mathrm{~N}-1$ & 9.07 & $\begin{array}{l}\text { Porous massive pyrite; colloform porous pyrite } \\
\text { aggregates in a matrix of pyrite and anhydrite. A single } \\
\text { large }(2.5 \mathrm{~cm}) \text { porous chalcopyrite fragment is probably a } \\
\text { chimney fragment. }\end{array}$ & anh & $\begin{array}{l}\text { Primary and secondary fluid inclusions in coarse- } \\
\text { grained matrix anhydrite cementing pyrite and } \\
\text { chalcopyrite }\end{array}$ & .9 & $242-295$ & 265 & 17.2 & 4 & $-1.8-2.9$ & -2.3 & 0.5 & 3.8 \\
\hline \multirow[t]{3}{*}{$5 \mathrm{~N}-1,73-75$} & \multirow[t]{3}{*}{27.35} & \multirow{3}{*}{$\begin{array}{l}\text { Nodular pyrite-silica breccia; large subrounded siliceous } \\
\text { fragments and nodular pyrite aggregates in a matrix of } \\
\text { fine-grained pyrite and quartz with minor vein-related } \\
\text { anhydrite. }\end{array}$} & qtz & $\begin{array}{l}\text { Primary fluid inclusions in a large quartz fragment and } \\
\text { dark siliceous matrix; secondary fluid inclusions in a }\end{array}$ & 27 & $302-339$ & 324 & 8.4 & 10 & $-2.6-3.7$ & -3.0 & 0.4 & 4.9 \\
\hline & & & & large quart & 13 & $262-308$ & 284 & 16.3 & 3 & $-2.6-2.9$ & -2.8 & 0.2 & 4.6 \\
\hline & & & anh & $\begin{array}{l}\text { Primary and secondary fluid inclusions in coarse- } \\
\text { grained, vein-related anhydrite. }\end{array}$ & 13 & $311-335$ & 319 & 6.3 & 4 & $-2.1-2.4$ & -2.3 & 0.1 & 3.9 \\
\hline $5 \mathrm{~N}-2,75-80$ & 28.17 & $\begin{array}{l}\text { Silicified wallrock breccia; large siliceous clast with } \\
\text { disseminated pyrite and stained by Fe-oxyhydroxides. }\end{array}$ & qtz & $\begin{array}{l}\text { Fluid inclusions at grain boundaries and primary } \\
\text { rounded inclusions in milky and transparent quartz, } \\
\text { secondary irregular inclusions in transparent quartz. }\end{array}$ & $\begin{array}{r}28 \\
4\end{array}$ & $\begin{array}{l}303-349 \\
284-295\end{array}$ & $\begin{array}{l}326 \\
290\end{array}$ & $\begin{array}{r}11.2 \\
4.8\end{array}$ & $\begin{array}{l}5 \\
1\end{array}$ & $\begin{array}{c}-2.7-2.9 \\
-\end{array}$ & $\begin{array}{l}-2.8 \\
-3.2\end{array}$ & $\begin{array}{l}0.1 \\
-\end{array}$ & $\begin{array}{l}4.8 \\
5.3\end{array}$ \\
\hline $8 \mathrm{~N}-1,77-79$ & 40.98 & $\begin{array}{l}\text { Pyrite-silica breccia; angular pyrite aggregates and } \\
\text { siliceous clasts in a dark siliceous matrix. Hematite and } \\
\text { anhydrite are filling vugs. }\end{array}$ & anh & $\begin{array}{l}\text { Primary angular to subrounded inclusions in coarse- } \\
\text { grained late anhydrite. }\end{array}$ & 9 & $337-346$ & 340 & 3.0 & 4 & $-2.7-3.0$ & -2.9 & 0.1 & 4.7 \\
\hline \multirow[t]{2}{*}{$\begin{array}{l}\text { TAG-4 } \\
158-957 \mathrm{M}- \\
2 \mathrm{R}-1,29-32\end{array}$} & \multirow[t]{2}{*}{9.59} & \multirow{2}{*}{$\begin{array}{l}\text { Pyrite-silica breccia; large angular gray siliceous clasts } \\
\text { with disseminated pyrite in a dark siliceous matrix } \\
\text { containing pyrite aggregates and disseminated pyrite. } \\
\text { Late marcasite/pyrite is lining vugs. }\end{array}$} & qtz & $\begin{array}{l}\text { Primary and } \\
\text { secondary inclusions in gray clasts with fine-grained } \\
(<100 \mu \mathrm{m}) \text { zoned euhedral quartz grains. }\end{array}$ & $\begin{array}{l}15 \\
14\end{array}$ & $\begin{array}{l}293-343 \\
198-293\end{array}$ & $\begin{array}{l}322 \\
248\end{array}$ & $\begin{array}{l}15.1 \\
29.5\end{array}$ & 二 & $\overline{-}$ & $\overline{-}$ & $\overline{-}$ & - \\
\hline & & & qtz & $\begin{array}{l}\text { Primary and } \\
\text { secondary inclusions in cryptocrystalline, often gel-like } \\
\text { quartz matrix with few euhedral quartz grains. }\end{array}$ & $\begin{array}{r}12 \\
5\end{array}$ & $\begin{array}{l}283-331 \\
257-313\end{array}$ & $\begin{array}{l}314 \\
286\end{array}$ & $\begin{array}{l}17.6 \\
24.8\end{array}$ & 二 & $\overline{-}$ & - & 二 & 二 \\
\hline 3R-1, 130-133 & 15.60 & $\begin{array}{l}\text { Pyrite-silica breccia; dark siliceous matrix containing } \\
\text { pyrite aggregates and few tiny gray siliceous clasts. Late } \\
\text { marcasite/pyrite is lining vugs. }\end{array}$ & qtz & $\begin{array}{l}\text { Primary and } \\
\text { secondary fluid inclusions in dark siliceous matrix } \\
\text { consisting of cryptocrystalline, often gel-like quartz } \\
\text { with few euhedral grains. }\end{array}$ & $\begin{array}{l}6 \\
5\end{array}$ & $\begin{array}{l}332-364 \\
262-319\end{array}$ & $\begin{array}{l}351 \\
288\end{array}$ & $\begin{array}{l}13.7 \\
21.1\end{array}$ & $\overline{-}$ & - & $\overline{-}$ & $\overline{-}$ & - \\
\hline $7 \mathrm{R}-1,0-3$ & 34.30 & $\begin{array}{l}\text { Gray quartz: small fragment of coarse-grained } \\
\text { recrystallized quartz stained by Fe-oxyhydroxides and } \\
\text { overgrown by pyrite. }\end{array}$ & qtz & $\begin{array}{l}\text { Primary and } \\
\text { secondary inclusions in transparent quartz. }\end{array}$ & $\begin{array}{l}2 \\
5\end{array}$ & $\begin{array}{l}327-363 \\
282-306\end{array}$ & $\begin{array}{l}345 \\
295\end{array}$ & $\begin{array}{r}25.5 \\
9.8\end{array}$ & $\begin{array}{l}1 \\
2\end{array}$ & $\begin{array}{c}-2.8 \\
-2.8-2.8\end{array}$ & $\begin{array}{l}02.8 \\
-2.8\end{array}$ & $\overline{-}$ & $\begin{array}{l}4.6 \\
4.6\end{array}$ \\
\hline
\end{tabular}


Table 1 (continued)

\begin{tabular}{|c|c|c|c|c|c|c|c|c|c|c|c|c|c|}
\hline \multirow{2}{*}{$\begin{array}{l}\text { Core, section, } \\
\text { interval }(\mathrm{cm})\end{array}$} & \multirow{2}{*}{$\begin{array}{l}\text { Depth } \\
\text { (mbsf) }\end{array}$} & \multirow[b]{2}{*}{ Sample type, description } & \multirow[b]{2}{*}{ Host } & \multirow[b]{2}{*}{ Comments } & \multirow[b]{2}{*}{$\mathrm{n}$} & \multicolumn{3}{|c|}{ Th $\left({ }^{\circ} \mathrm{C}\right)$} & \multirow[b]{2}{*}{$\mathrm{n}$} & \multicolumn{3}{|c|}{$\operatorname{Tm}\left({ }^{\circ} \mathrm{C}\right)$} & \multirow{2}{*}{$\begin{array}{l}\mathrm{NaCl} \\
\mathrm{wt} \%\end{array}$} \\
\hline & & & & & & Range & Average & SD & & Range & Average & SD & \\
\hline 10R-1, 123-125 & 47.22 & $\begin{array}{l}\text { Gray quartz: fine-grained quartz weakly stained by Fe- } \\
\text { oxyhydroxides and containing a chloritized basalt } \\
\text { fragment. The sample is partly rimmed by pyrite. }\end{array}$ & qtz & $\begin{array}{l}\text { Primary and } \\
\text { secondary fluid inclusions in fine-grained, zoned, } \\
\text { euhedral quartz }(<100 \mu \mathrm{m}) \text {. }\end{array}$ & $\begin{array}{l}5 \\
8\end{array}$ & $\begin{array}{l}250-271 \\
191-233\end{array}$ & $\begin{array}{l}256 \\
220\end{array}$ & $\begin{array}{r}9.0 \\
14.3\end{array}$ & 1 & 二 & -3.7 & 二 & ${ }^{6.0}$ \\
\hline \multicolumn{14}{|l|}{$\begin{array}{l}\text { TAG-5 } \\
158-957 \mathrm{P}\end{array}$} \\
\hline $5 \mathrm{R}-1,9-13$ & 21.57 & $\begin{array}{l}\text { Massive granular pyrite: massive pyrite with minor } \\
\text { chalcopyrite from pyritization halo with vein-related } \\
\text { anhydrite rim. }\end{array}$ & anh & $\begin{array}{l}\text { Primary, angular fluid inclusions in medium-grained } \\
\text { anhydrite cementing pyrite aggregates. }\end{array}$ & 10 & $283-302$ & 292 & 6.7 & 6 & $-2.4-2.8$ & -2.6 & 0.1 & 4.4 \\
\hline $9 \mathrm{R}-1,1-3$ & 40.10 & $\begin{array}{l}\text { Nodular pyrite-silica breccia: light gray fine-grained } \\
\text { siliceous matrix with disseminated pyrite containing } \\
\text { millimeter- to centimeter-sized rounded pyrite } \\
\text { aggregates. }\end{array}$ & qtz & $\begin{array}{l}\text { Primary and } \\
\text { secondary fluid inclusions in fine-grained, milky quartz } \\
\text { matrix with euhedral medium-grained quartz growing } \\
\text { into void space. }\end{array}$ & $\begin{array}{r}8 \\
12\end{array}$ & $\begin{array}{l}322-351 \\
234-316\end{array}$ & $\begin{array}{l}337 \\
277\end{array}$ & $\begin{array}{l}10.6 \\
18.7\end{array}$ & $\overline{1}$ & 二 & $\overline{-3.2}$ & 二 & $\overline{5.3}$ \\
\hline $12 \mathrm{R}-2,25-28$ & 55.89 & $\begin{array}{l}\text { Pyrite-silica breccia: gray quartz with disseminated } \\
\text { medium-grained pyrite and anhydrite filling voids. }\end{array}$ & anh & $\begin{array}{l}\text { Primary subangular, subrounded and needle shaped fluid } \\
\text { inclusions in coarse-grained anhydrite (up to } 1 \mathrm{~mm} \text { ). }\end{array}$ & 15 & $343-357$ & 352 & 4.6 & 4 & $-2.8-3.1$ & -3.0 & 0.1 & 4.9 \\
\hline $12 \mathrm{R}-4,35-37$ & 57.44 & $\begin{array}{l}\text { Silicified wallrock breccia: dark gray strongly silicified } \\
\text { wallrock fragments. Igneous textures are still } \\
\text { recognizable. Sample is rimmed by vein-related pyrite. }\end{array}$ & qtz & $\begin{array}{l}\text { Primary and } \\
\text { secondary fluid inclusions in medium-grained quartz } \\
\text { related to quartz-pyrite veins crosscutting wallrock } \\
\text { fragments; matrix quartz is characterized by abundant, } \\
\text { small }(<1 \mu \mathrm{m}) \text { inclusions. }\end{array}$ & $\begin{array}{l}9 \\
9\end{array}$ & $\begin{array}{l}317-356 \\
271-321\end{array}$ & $\begin{array}{l}338 \\
306\end{array}$ & $\begin{array}{l}15.4 \\
11.3\end{array}$ & $\begin{array}{l}1 \\
1\end{array}$ & 二 & $\begin{array}{l}-2.8 \\
-3.0\end{array}$ & 二 & $\begin{array}{l}4.6 \\
5.0\end{array}$ \\
\hline
\end{tabular}

Note: anh $=$ anhydrite; qtz = quartz; $-=$ not measured. 


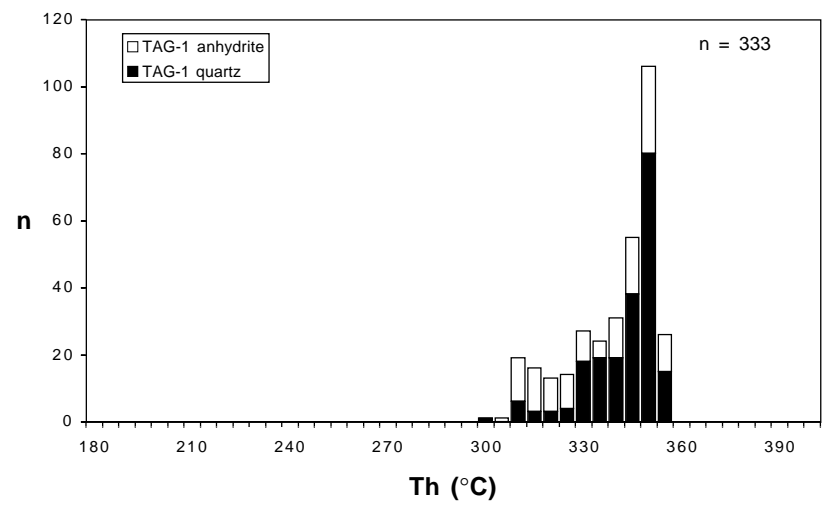

Figure 4. Frequency histogram of homogenization temperatures (Th) for fluid inclusions in quartz and anhydrite from the TAG-1 area, close to the Black Smoker Complex. $\mathrm{n}=$ number of analyses.

ed near the bottom of the hole (Sample 158-957H-8N-1, 77-79 cm; $41 \mathrm{mbsf}$ ) exhibits homogenization temperatures of $337^{\circ}-346^{\circ} \mathrm{C}$ (avg $=340^{\circ} \mathrm{C}, \mathrm{n}=9$ ). The strong increase of pressure-corrected temperatures with depth is evident from Figure 7A.

The western side of the mound (TAG-4) is characterized by a broad variation of homogenization temperatures ranging from $191^{\circ}$ to $364^{\circ} \mathrm{C}$ (Fig. $\left.6 \mathrm{~B}\right)$. The average of $289^{\circ} \mathrm{C}(\mathrm{SD}=44.0, \mathrm{n}=77)$ is much lower than results from other areas. A pyrite-silica breccia from the uppermost part of the mound (Sample 158-957M-2R-1, 29-32 cm) is characterized by light gray siliceous clasts in a dark gray siliceous matrix. Homogenization temperatures in these early clasts and the siliceous matrix are very similar (see Table 1). Primary fluid inclusions in the siliceous clasts exhibit homogenization temperatures of $293^{\circ}-343^{\circ} \mathrm{C}$ with an average of $322^{\circ} \mathrm{C}(\mathrm{n}=15)$, whereas homogenization temperatures for primary inclusions in the siliceous matrix range from $283^{\circ}$ to $331^{\circ} \mathrm{C}\left(\right.$ avg $\left.=314^{\circ} \mathrm{C}, \mathrm{n}=12\right)$. Homogenization temperatures for secondary inclusions in the early clasts and the matrix are much lower and range from $198^{\circ}$ to $293^{\circ} \mathrm{C}\left(\operatorname{avg}=248^{\circ} \mathrm{C} ; \mathrm{n}=\right.$ 14) in the quartz clast and from $257^{\circ}$ to $313^{\circ} \mathrm{C}\left(\mathrm{avg}=286^{\circ} \mathrm{C}, \mathrm{n}=5\right.$ ) in the siliceous matrix. Two pyrite-silica breccias taken deeper downcore show homogenization temperatures of primary fluid inclusions that are much higher and range from $332^{\circ}-364^{\circ} \mathrm{C}\left(\mathrm{avg}=350^{\circ} \mathrm{C}, \mathrm{n}=\right.$ 8; Samples 158-957M-3R-1, 130-133 cm and 158-957M-7R-1, 0-3 $\mathrm{cm})$. Secondary inclusions in these samples show similar homogenization temperatures when compared to secondary inclusions in samples collected near the surface and range from $262^{\circ}-319^{\circ} \mathrm{C}$ (avg = $292^{\circ} \mathrm{C}, \mathrm{n}=10$ ). A strong decrease in homogenization temperatures occurs in a gray chert sample, associated with greenish, strongly chloritized basalt fragments, taken at 47.22 mbsf near the bottom of Hole $957 \mathrm{M}$. Primary fluid inclusions in quartz are characterized by homogenization temperatures in the range from $250^{\circ}$ to $271^{\circ} \mathrm{C}$ (avg $=256^{\circ} \mathrm{C}, \mathrm{n}=5$ ), whereas secondary inclusions show lower homogenization temperatures in the range from $191^{\circ}$ to $233^{\circ} \mathrm{C}$ (avg $=220^{\circ} \mathrm{C}$, $\mathrm{n}=8$; Sample 158-957M-10R-1, 123-125 cm). Trapping temperatures in the TAG-4 area vary from $212^{\circ}$ to $390^{\circ} \mathrm{C}(\mathrm{n}=77)$. The downhole distribution of pressure-corrected homogenization temperatures at TAG-4 is given in Fig. 7B.

Samples from the northern side of the mound (TAG-5) exhibit homogenization temperatures in the range from $234^{\circ}$ to $357^{\circ} \mathrm{C}(\operatorname{avg}=$ $318^{\circ} \mathrm{C}, \mathrm{SD}=31.3, \mathrm{n}=61$; Fig $6 \mathrm{C}$ ). Anhydrite cementing massive granular pyrite in the upper part of TAG-5 (above $25 \mathrm{mbsf}$ ) is characterized by homogenization temperatures ranging from $283^{\circ}$ to $302^{\circ} \mathrm{C}\left(\right.$ avg $=292^{\circ} \mathrm{C}, \mathrm{n}=8$, Sample 158-957P-5R-1, 9-13 cm). In contrast, anhydrite rimming a pyrite-silica breccia in the lower part of TAG- 5 shows a narrow range of much higher values between $343^{\circ}$ and $357^{\circ} \mathrm{C}\left(\operatorname{avg}=352^{\circ} \mathrm{C}, \mathrm{n}=15\right.$, Sample 158-957P-12R-2, 25-28 $\mathrm{cm})$. Primary fluid inclusions in quartz from a pyrite-silica breccia from the center of the mound are characterized by homogenization temperatures ranging from $322^{\circ}$ to $351^{\circ} \mathrm{C}$ (avg $=337^{\circ} \mathrm{C}, \mathrm{n}=8$, Sample 158-957P-9R-1, 1-3 cm). Secondary fluid inclusions show much lower values in the range from $234^{\circ}$ to $316^{\circ} \mathrm{C}$ with an average of $277^{\circ} \mathrm{C}(\mathrm{n}=12)$. Homogenization temperatures of quartz from a silicified wallrock breccia from the bottom of the hole are very similar to those of the pyrite-silica breccia above. Primary inclusions show homogenization temperatures between $317^{\circ}$ and $356^{\circ} \mathrm{C}\left(\right.$ avg $=338^{\circ} \mathrm{C}$, $\mathrm{n}=9$, Sample 158-957P-12R-4, 35-37 cm), whereas secondary inclusions display variations from $271^{\circ}$ to $321^{\circ} \mathrm{C}\left(\mathrm{avg}=306^{\circ} \mathrm{C}, \mathrm{n}=9\right.$ ). The vertical temperature increase in this area is evident when pressure corrected temperatures are plotted against depth (Fig. 7C).

The salinity variation with depth in areas TAG-1, TAG-2, and TAG-5 is given in Figure 8 and shows no clear trend to higher salinities with depth in any drilling area, but the salinity variations differ from area to area. While the TAG-1 area exhibits the whole range of salinities observed at TAG (1.9 to $6.2 \mathrm{wt} \% \mathrm{NaCl}$ equivalent, avg = $4.4 \mathrm{wt} \% \mathrm{NaCl}$ equivalent, $\mathrm{SD}=1.0, \mathrm{n}=77)$, the salinity variations for areas TAG-2 (3.1 to $6.0 \mathrm{wt} \% \mathrm{NaCl}$ equivalent, avg $=4.6 \mathrm{wt} \%$ $\mathrm{NaCl}$ equivalent, $\mathrm{SD}=0.6, \mathrm{n}=31)$ and TAG-5 $(4.0$ to $5.3 \mathrm{wt} \% \mathrm{NaCl}$ equivalent, avg $=4.7 \mathrm{wt} \% \mathrm{NaCl}$ equivalent, $\mathrm{SD}=0.4, \mathrm{n}=13$ ) are much smaller. There are, so far, only limited data for fluid inclusions in the TAG-4 area, where salinities of 2.8 and $3.7 \mathrm{wt} \% \mathrm{NaCl}$ equivalent have been measured $(n=4)$.

\section{DISCUSSION}

Fluid inclusion measurements of quartz and anhydrite in various breccias from the active TAG hydrothermal mound at the Mid-Atlantic Ridge indicate high-temperature formation of most parts of the TAG mound at one time during the development of the system. Fluid inclusion measurements in quartz and anhydrite reveal differences in hydrothermal flow within the mound during subsequent hydrothermal cycles. While quartz represents both old and young hydrothermal activity, anhydrite is believed to be the result of the most recent hydrothermal episode. It has been shown that hydrothermal activity at TAG is intermittent, with high-temperature hydrothermal pulses separated by periods of quiescence (Lalou et al., 1995). Anhydrite has probably been totally dissolved during these periods, because of its retrograde solubility in seawater at temperatures below $150^{\circ} \mathrm{C}$.

Trapping temperatures for fluid inclusions in anhydrite samples just underlying the presently venting Black Smoker Complex (TAG1) and the Kremlin area (TAG-2) are very similar to the exit temperatures of the vents in these areas. The anhydrite zone observed at 20 to $35 \mathrm{mbsf}$ in the TAG-1 area shows trapping temperatures of $340^{\circ}$ $360^{\circ} \mathrm{C}$, which is slightly lower than the exit temperature of hydrothermal fluids presently venting at the Black Smoker Complex $\left(360^{\circ}\right.$ $366^{\circ} \mathrm{C}$; Edmond et al., 1995). These results are also in good agreement with fluid inclusion measurements performed on massive anhydrite from the base of the Black Smoker Complex, where trapping temperatures of $340^{\circ}-350^{\circ} \mathrm{C}$ have been found (Hannington et al., 1995; Tivey et al., Chap. 14, this volume). Fluid inclusions from anhydrite in samples collected near the surface of the Kremlin area (TAG-2) are characterized by trapping temperatures in the range from $266^{\circ}$ to $321^{\circ} \mathrm{C}$, which is comparable to the exit temperatures of the presently venting white smoker fluids $\left(270^{\circ}-300^{\circ} \mathrm{C}\right.$, Edmond et al., 1995). There is generally a good agreement between average trapping temperatures obtained from fluid inclusion measurements and the actual temperature of venting in the same hydrothermal systems (Le Bel and Oudin, 1982; Brett et al., 1987; Hannington and Scott, 1988; Peter and Scott, 1988; Leitch, 1991), although in most cases, except for Middle Valley (Peter et al., 1994), only surface samples 


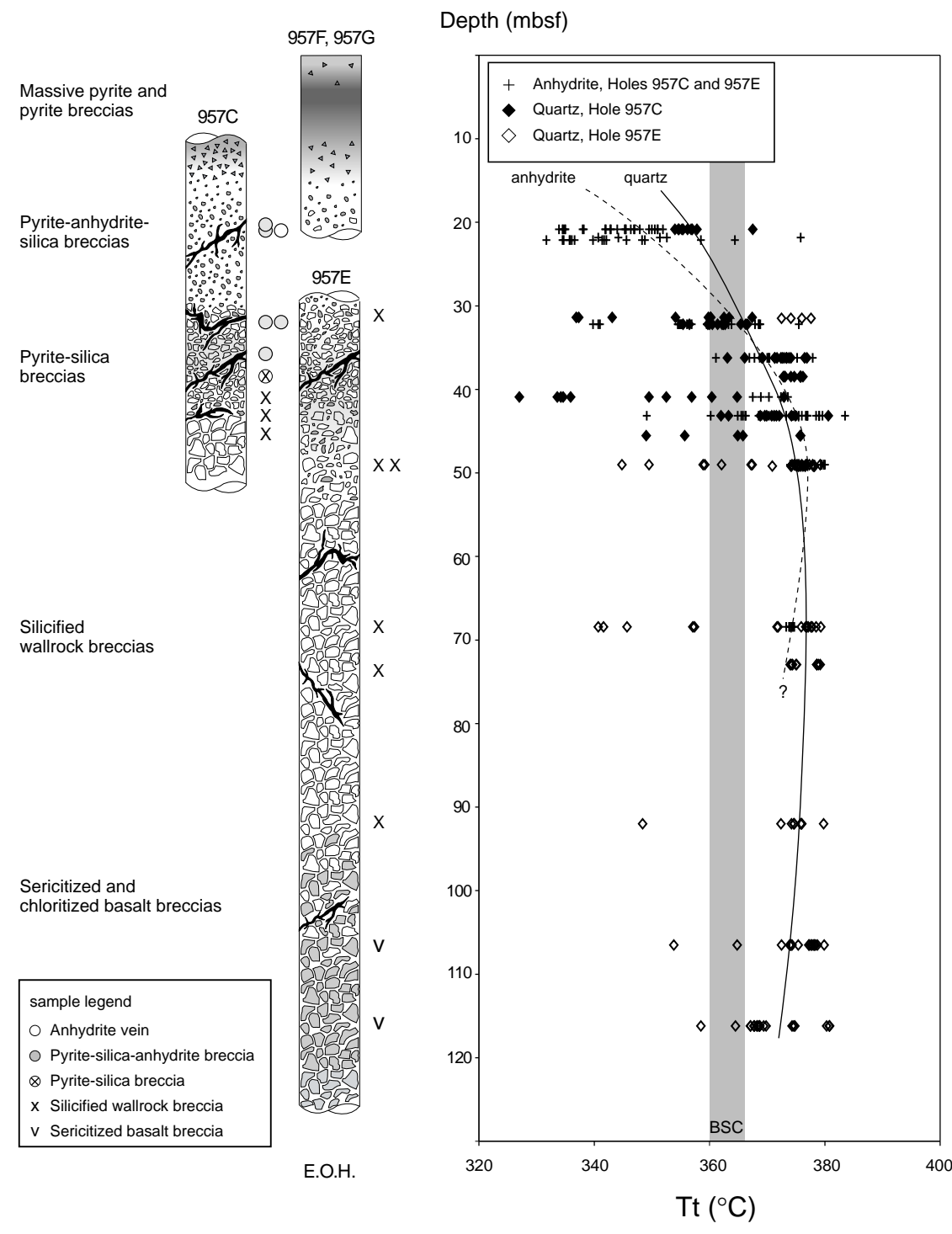

Figure 5. Vertical depth variation of trapping temperatures $(\mathrm{Tt})$ for fluid inclusions in quartz and anhydrite for the TAG-1 area. A simplified stratigraphy and the sample locations are also given. The lines for anhydrite and quartz outline the general increase of temperature with depth. The curves are hand drawn and represent a smoothed curve connecting averages for individual samples. The exit temperatures of the central Black Smoker Complex (BSC) are indicated by the gray box (Edmond et al., 1995). E.O.H. = end of hole. have been studied. Trapping temperatures in the stockwork zone underlying the Black Smoker Complex are generally higher than $375^{\circ} \mathrm{C}$. These temperatures are higher than the exit temperatures of fluids presently venting at the Black Smoker Complex and may indicate conductive cooling or mixing of the hydrothermal fluids with seawater before venting at the Black Smoker Complex. A strong temperature increase with depth has been observed in the Kremlin area and suggests a high-temperature stockwork zone similar to the TAG1 area, although the overall slightly lower trapping temperatures indicate enhanced mixing or cooling of the hydrothermal fluid.

Samples from the northern side of the mound (TAG-5) are lithologically similar to those from the Black Smoker Complex, although differences in chemistry and mineralogy occur (Herzig et al., Chap. 4, this volume; Hannington et al., Chap. 2, this volume; Knott et al., Chap. 1, this volume). Fluid inclusion measurements show a strong temperature increase with depth, similar to the Kremlin area. Anhydrite from the bottom of TAG-5 was formed at temperatures higher than preexisting quartz in this area. This recent temperature increase might be related to the formation of a depression at the northeastern side of the mound not far from the TAG-5 area observed by Kleinrock et al. (1996). This 20-m-wide, 25-m-long, and 10-m-deep depression trending north-northeast is emitting black smoker fluid and is probably related to recent tectonic activity in this area that is intensifying the heat flow and hydrothermal discharge distal to the central Black Smoker Complex. Other isolated occurrences of black smoker fluid have also been described by Kleinrock et al. (1996), testifying that the hydrothermal "end-member" upflow is not restricted to the central Black Smoker Complex.

Fluid inclusion measurements in areas TAG-1, TAG-2, and TAG5 have shown that the trapping temperatures and salinities of primary inclusions in anhydrite and quartz are comparable and show similar downhole trends in these areas. Secondary fluid inclusions in quartz in areas TAG-2, TAG-4, and TAG-5 indicate that these areas have been affected by later, lower temperature hydrothermal fluids, probably during the waning stages of hydrothermal activity. These secondary fluids have not been observed in the TAG-1 area close to the proposed hydrothermal upflow zone. Since both host minerals, quartz and anhydrite, show vary similar trapping temperatures, it can be argued that the hydrothermal upflow zone remained constant over time in this area.

The west side of the mound (TAG-4) is characterized by a coherent belt of very low heat flow (Becker and Von Herzen, 1996) that has been explained by local entrainment of seawater (Tivey et al., 1995). Humphris et al. (1995) have argued that the western side of the 
A

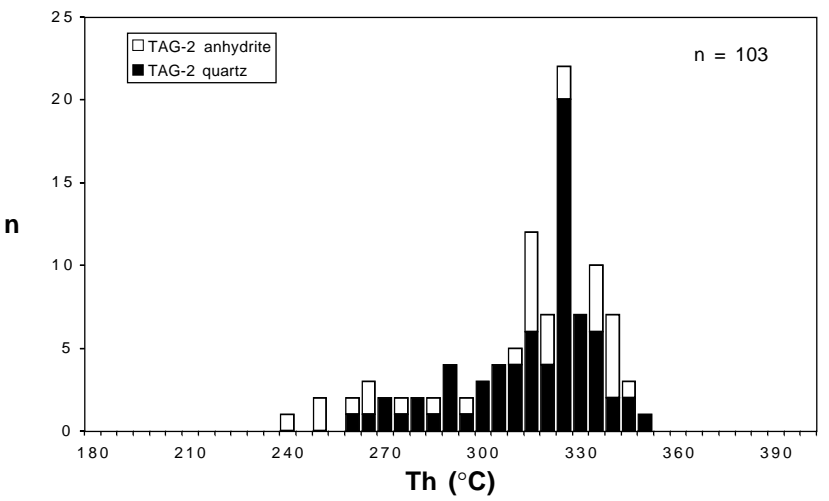

B
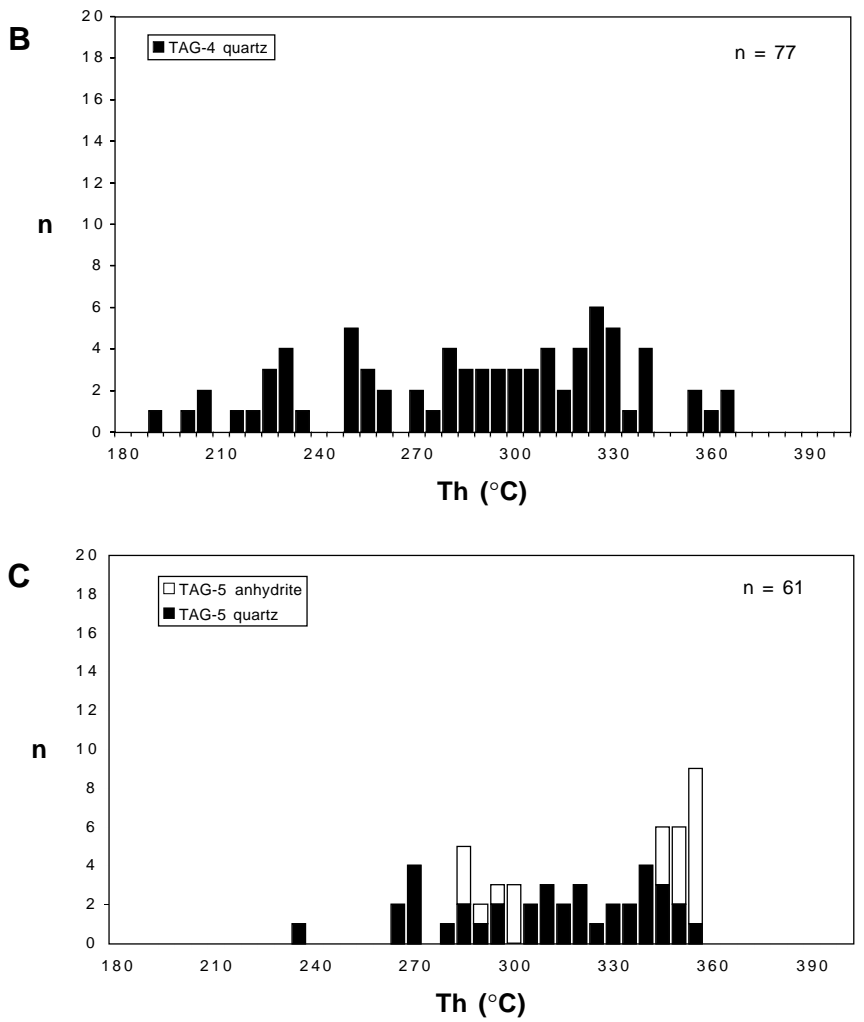

Figure 6. Frequency histogram of homogenization temperatures for fluid inclusions in quartz and anhydrite from (A) TAG-2, (B) TAG-4, and (C) TAG-5.

mound might not be part of the high-temperature stockwork at all, but instead related to a talus pile resulting from the mass wasting of hydrothermal precipitates higher up the mound that were subsequently silicified at distinctly lower temperatures than at the Black Smoker Complex. Their conclusion was derived from the absence of anhydrite, a strong silicification of basaltic clasts, and the freshness of the underlying basalt. Trapping temperatures for primary fluid inclusions in quartz from pyrite-silica breccias from the center of the mound, however, reach $390^{\circ} \mathrm{C}$, indicating that silicification in these samples was a high-temperature event. Lower trapping temperatures were found in a pyrite-silica breccia collected near the surface of the mound and from a sample collected near the bottom of the hole just overlying the weakly altered basalt. The average trapping temperature of $280^{\circ} \mathrm{C}(\mathrm{n}=5)$ for the latter is the lowest trapping temperature measured during this study, but even these values imply that this area has been part of a high-temperature hydrothermal upflow zone in the
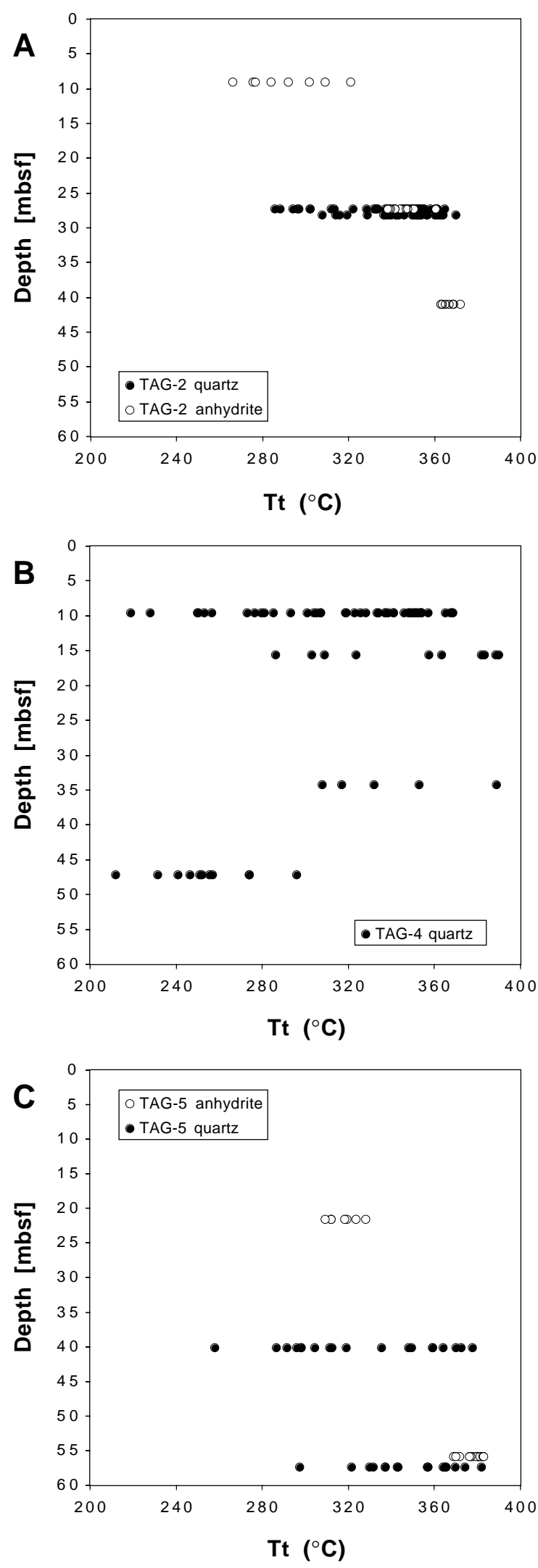

Figure 7. Vertical depth variation of trapping temperatures (Tt) for fluid inclusions in quartz and anhydrite from (A) the Kremlin area (TAG-2), (B) the west side of the mound (TAG-4), and (C) the northern side of the mound (TAG-5). 

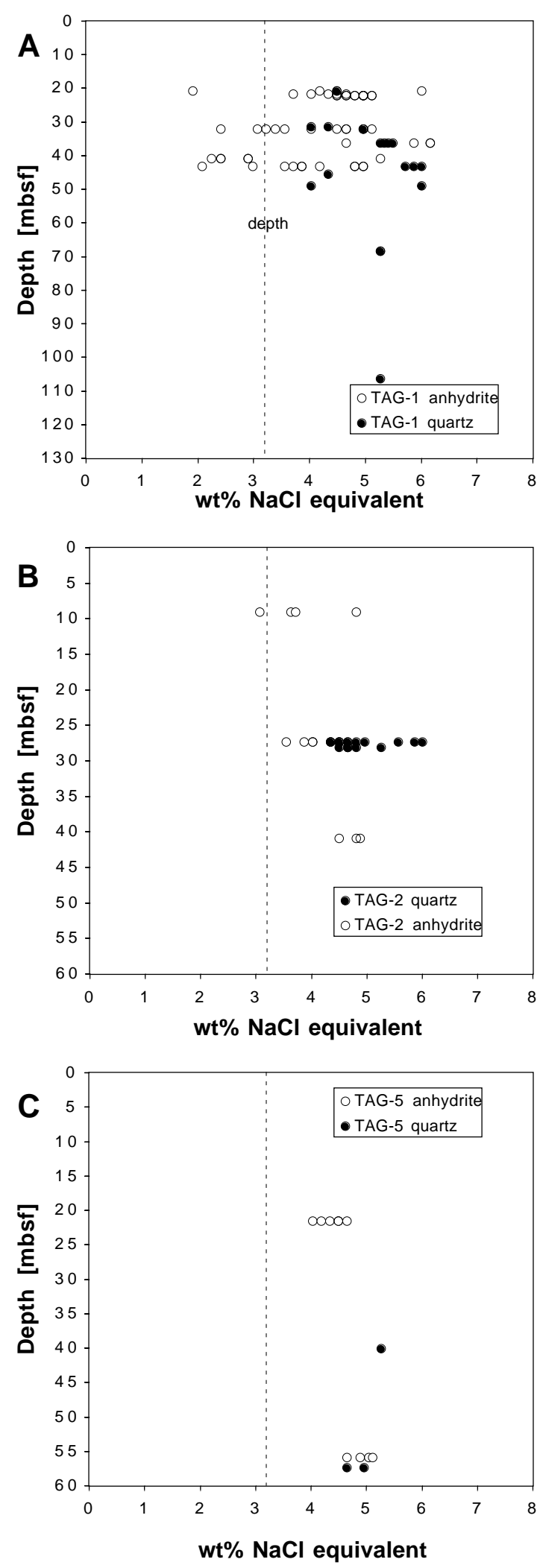

Figure 8. Vertical depth variation of salinities for fluid inclusions in quartz and anhydrite from (A) the Black Smoker Complex (TAG-1), (B) the Kremlin area (TAG-2), and (C) the northern side of the mound (TAG-5). The vertical line indicates the salinity of seawater. past. The average trapping temperatures for secondary fluid inclusions in samples from the TAG-4 area range from $243^{\circ}$ to $321^{\circ} \mathrm{C}$ and probably indicate circulation of slightly lower temperature hydrothermal fluids during the waning stage of hydrothermal activity in this area. The very low heat flow, the absence of venting at the surface, and the absence of anhydrite within the mound in the TAG-4 area are strong indications that the recent pulse of high-temperature hydrothermal activity is not affecting TAG-4.

The salinity of fluid inclusions in samples from the TAG mound varies from 0.6 to 2 times seawater ( $3.2 \mathrm{wt} \% \mathrm{NaCl}$ equivalent). Similar salinity variations in fluid inclusions have been found in samples from other mid-ocean ridge sites (Nehlig, 1991; Peter et al., 1994) and in samples from the upflow zone of these systems (Delaney et al., 1987; Vanko, 1988, Vanko et al., 1992; Kelley et al., 1993; Saccocia and Gillis, 1995). Hydrothermal fluids at mid-ocean ridge sites also display similar salinity variations, where values from $<10 \%$ to $>230 \%$ seawater occur (see summary in de Ronde [1995]). However, some hydrothermal systems seem to be characterized by strongly $\mathrm{Cl}-$ depleted vent fluids (Butterfield et al., 1994; Von Damm et al., 1995), whereas others are Cl-enriched (Von Damm and Bischoff, 1987; Butterfield and Massoth, 1994). Salinity variations have also been observed in quartz related to fossil massive sulfide deposits in the Troodos ophiolite in Cyprus and the Semail ophiolite in Oman (Spooner and Bray, 1977; Nehlig, 1991; Kelley et al., 1992). Several mechanisms have been proposed to explain the salinity variations in hydrothermal systems at mid-ocean ridges. Possible explanations include (1) hydration of the oceanic crust (Palmer, 1992); (2) precipitation and later dissolution of a Cl-bearing mineral during alteration (Seyfried et al., 1986; Vanko 1986); (3) phase separation of seawater derived fluids (Bischoff and Pitzer, 1985; Edmonds and Edmond, 1995); and (4) $\mathrm{H}_{2} \mathrm{O}$ - or Cl-rich magmatic fluids. The changes in the $\mathrm{Cl}$-concentration of hydrothermal fluids caused by the first two processes are thought to be small (Ito and Anderson, 1983; Berndt and Seyfried, 1990). Phase separation seems to be the only reasonable process to account for the wide ranges in salinity observed in vent fluids and in fluid inclusions from samples associated with deep circulating fluids or shallow hydrothermal systems. Both subcritical (boiling) and supercritical phase separation (i.e. condensation of a small amount of highly saline liquid) have been suggested as possible processes (Von Damm and Bischoff, 1987; Kelley and Delaney, 1987; Cowen and Cann, 1988; Von Damm, 1990; Butterfield et al., 1990).

The depth of the TAG hydrothermal mound $(3650 \mathrm{~m})$ requires that phase separation took place above the critical point of seawater $\left(407^{\circ} \mathrm{C}, 298\right.$ bar; Bischoff and Rosenbauer, 1988). Therefore, supercritical phase separation of a magmatic or seawater-derived fluid with partial segregation of the liquid and the vapor phase at depth, followed by partial mixing of the brine and the vapor phase during ascent to shallower crustal levels may explain the salinity differences observed in samples from the TAG mound. Similar processes have been used to explain salinity variations in samples from the MARK area (Delaney et al., 1987; Kelley et al., 1993) and the Hess Deep (Saccocia and Gillis, 1995). The conditions under which phase separation probably occurred can be illustrated in an isothermal (P-X) projection of the $\mathrm{NaCl}-\mathrm{H}_{2} \mathrm{O}$ system (Fig. 9; after Bischoff and Pitzer, 1989). Isotherms define the boundary between the one-phase field and the two-phase field. The lowest salinity observed in samples from the TAG mound ( $1.2 \mathrm{wt} \% \mathrm{NaCl}$ equivalent) has been reported by Tivey et al. (Chap. 14, this volume) and expands the range for reported salinities to 1.2 to $6.2 \mathrm{wt} \% \mathrm{NaCl}$ equivalent. If we assume that the minimum depth at which phase separation occurred is just below the termination of Hole 957E (3772 m), than the minimum temperature at which phase separation could have occurred at TAG is $\sim 440^{\circ} \mathrm{C}$. Phase separation of a hydrothermal fluid of seawater salinity at this temperature would produce a vapor phase with a salinity of 2.0 $\mathrm{wt} \% \mathrm{NaCl}$ equivalent and a liquid phase with a salinity of $15 \mathrm{wt} \%$ $\mathrm{NaCl}$ equivalent. The salinity of the vapor phase is higher than the 


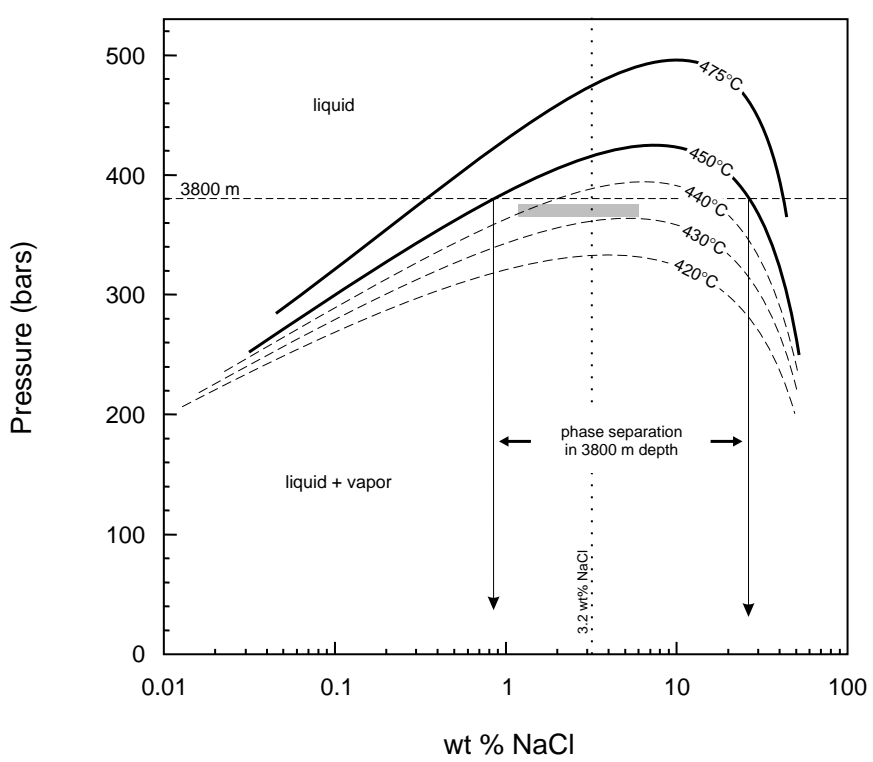

Figure 9. Isothermal $\mathrm{P}-\mathrm{X}$ projection of the system $\mathrm{NaCl}-\mathrm{H}_{2} \mathrm{O}$ under hydrostatic conditions (after Bischoff and Pitzer, 1989). In order to produce the salinity variation observed in fluid inclusions at TAG (stippled area), a seawater-derived fluid has to intersect the two-phase boundary at temperatures above $450^{\circ} \mathrm{C}$. Isotherms that do not apply at TAG are shown as dashed lines. See text for details.

lowest salinity observed at TAG and can therefore not explain the salinity variation at TAG. In order to produce the low-salinity fluids at TAG, the minimum temperature at which phase separation occurred has to be close to $450^{\circ} \mathrm{C}$ and would produce a vapor phase with a salinity of $0.9 \mathrm{wt} \% \mathrm{NaCl}$ equivalent and a brine phase with a salinity of $25 \mathrm{wt} \% \mathrm{NaCl}$ equivalent, which is much higher than the highest salinity observed at TAG. Two possible mechanisms could explain the lower range of salinities. Because of the relatively small density difference between coexisting liquids and vapors at these temperatures and pressures, the liquid phase will probably partially ascend together with the vapor phase and remix during the ascent (Bischoff and Rosenbauer, 1987). Mixing of the hydrothermal fluids with various amounts of seawater within the mound will further narrow the range of salinities. A second explanation might be offered by the episodic release of brines from the underlying plutonic sequence into the overlying hydrothermal systems as suggested by Kelley et al. (1993) for the MARK area. This process could also produce fluctuations of salinities with time. The fact that quartz is solely characterized by enriched salinities might be explained by formation of quartz during the culmination of hydrothermal activity and episodic release of brines into the hydrothermal system.

\section{CONCLUSIONS}

Fluid inclusion studies in quartz and anhydrite from various lithologies drilled during Leg 158 reveal the recent and past temperature regime within the active TAG mound. Trapping temperatures are generally high, but show a strong vertical and horizontal variation between different areas of the mound. Anhydrite probably represents recent hydrothermal activity, whereas quartz has been formed during recent and past hydrothermal activity. Trapping temperatures for anhydrite collected just below the actively venting Black Smoker Complex and the Kremlin area are consistent with measured exit temperatures of hydrothermal fluids in these areas. The occurrence of anhy- drite that formed at high temperatures in the northern part of the mound indicates that upflow of black smoker fluids is not restricted to the Black Smoker Complex. A strong vertical temperature increase indicates temperatures in excess of $380^{\circ} \mathrm{C}$ in the lower stockwork of the mound. Microthermometric results for samples from the area with low heat flow to the west of the Black Smoker Complex indicate that past high-temperature hydrothermal fluids have affected this area, despite its present inactivity. The range of salinities that has been observed at TAG is similar to those found in fluid inclusions and vent fluids from other mid-ocean ridge hydrothermal systems and is best explained by supercritical phase separation at temperatures above $450^{\circ} \mathrm{C}$ and subsequent remixing of vapor and liquid phase during the ascent, probably with some brine loss at depth.

\section{ACKNOWLEDGMENTS}

This work was supported through a grant of the Deutsche Forschungsgemeinschaft (DFG grant No. He 1660/5) and the NATO Scientific Affairs Division (grant No. CRG 941218). We thank the crew of the JOIDES Resolution for their effort to obtain samples in the difficult environment of the TAG mound. This work is part of an ongoing Ph.D. project of SP at Freiberg University of Mining and Technology. Thorough reviews by P. Nehlig and especially D. Kelley greatly improved the original manuscript.

\section{REFERENCES}

Becker, K., and Von Herzen, R.P., 1996. Pre-drilling observations of conductive heat flow at the TAG active mound using Alvin. In Humphris, S.E., Herzig, P.M., Miller, D.J., et al., Proc. ODP, Init. Repts., 158: College Station, TX (Ocean Drilling Program), 23-29.

Berndt, M.E., and Seyfried, W.E., Jr., 1990. Boron, bromine, and other trace elements as clues to the fate of chlorine in mid-ocean ridge vent fluids. Geochim. Cosmochim. Acta, 54:2235-2245.

Bischoff, J.L., and Pitzer, K.S., 1985. Phase relations and adiabats in boiling seafloor geothermal systems. Earth Planet. Sci. Lett., 75:327-338.

, 1989. Liquid-vapor relations for the system $\mathrm{NaCl}-\mathrm{H}_{2} \mathrm{O}$ : summary of the P-T-x surface from $300^{\circ}$ to $500^{\circ} \mathrm{C}$. Am. J. Sci., 289:217-248.

Bischoff, J.L., and Rosenbauer, R.J., 1988. Liquid-vapor relations in the critical region of the system $\mathrm{NaCl}-\mathrm{H}_{2} \mathrm{O}$ from $380^{\circ} \mathrm{C}$ to $415^{\circ} \mathrm{C}$ : a refined determination of the critical point and two-phase boundary of seawater. Geochim. Cosmochim. Acta, 52:2121-2126.

Blount, C.N., and Dickson, F.W., 1969. The solubility of anhydrite $\left(\mathrm{CaSO}_{4}\right)$ in $\mathrm{NaCl}-\mathrm{H}_{2} \mathrm{O}$ from 100 to $450^{\circ} \mathrm{C}$ and 1 to 100 bars. Geochim. Cosmochim. Acta, 33:227-245.

Bodnar, R.J., 1993. Revised equation and table for determining the freezing point depression of $\mathrm{H}_{2} \mathrm{O}-\mathrm{NaCl}$ solutions. Geochim. Cosmochim. Acta, 57:683-684.

Brett, R., Evans, H.T., Jr., Gibson, E.K., Jr., Hedenquist, J.W., Wandless, M.V., and Sommer, M.A., 1987. Mineralogical studies of sulfide samples and volatile concentrations of basalt glasses from the southern Juan de Fuca Ridge. J. Geophys. Res., 92:11,373-11,379.

Butterfield, D.A., and Massoth, G.J., 1994. Geochemistry of north Cleft segment vent fluids: temporal changes in chlorinity and their possible relation to recent volcanism. J. Geophys. Res., 99:4951-4968.

Butterfield, D.A., Massoth, G.J., McDuff, R.E., Lupton, J.E., and Lilley, M.D., 1990. Geochemistry of hydrothermal fluids from Axial Seamount Hydrothermal Emissions Study vent field, Juan de Fuca Ridge: subseafloor boiling and subsequent fluid-rock interaction. J. Geophys. Res., 95:12,895-12,921.

Butterfield, D.A., McDuff, R.E., Mottl, M.J., Lilley, M.D., Lupton, J.E., and Massoth, G.J., 1994. Gradients in the composition of hydrothermal fluids from the Endeavour segment vent field: phase separation and brine loss. J. Geophys. Res., 99:9561-9583.

Campbell, A.C., Palmer, M.R., Klinkhammer, G.P., Bowers, T.S., Edmond, J.M., Lawrence, J.R., Casey, J.F., Thompson, G., Humphris, S., Rona, P.A., and Karson, J.A., 1988. Chemistry of hot springs on the Mid-Atlantic Ridge. Nature, 335:514-519. 
Cowan, J., and Cann, J., 1988. Supercritical two-phase separation of hydrothermal fluids in the Troodos ophiolite. Nature, 333:259-261.

Davis, E.E., Mottl, M.J., Fisher, A.T., et al., 1992. Proc. ODP, Init. Repts., 139: College Station, TX (Ocean Drilling Program).

Delaney, J.R., Mogk, D.W., and Mottl, M.J., 1987. Quartz-cemented breccias from the Mid-Atlantic Ridge: samples of a high-salinity hydrothermal upflow zone. J. Geophys. Res., 92:9175-9192.

de Ronde, C.E.J., 1995. Fluid chemistry and isotope characteristics of seafloor hydrothermal systems and associated VMS deposits: potential for magmatic contributions. In Thompson, J.F.H. (Ed.), Magmas, Fluids, and Ore Deposits. Mineral. Assoc. Can. Short Course, 23:479-509.

Edmond, J.M., Campbell, A.C., Palmer, M.R., German, C.R., Klinkhammer, G.P., Edmonds, H.N., Elderfield, H., Thompson, G., and Rona, P., 1995. Time series studies of vent fluids from the TAG and MARK sites (1986, 1990): Mid-Atlantic Ridge: a new solution chemistry model and a mechanism for $\mathrm{Cu} / \mathrm{Zn}$ zonation in massive sulfide ore bodies. In Parson, L.M., Walker, C.L., and Dixon, D.R. (Eds.), Hydrothermal Vents and Processes. Geol. Soc. Spec. Publ. London, 87:77-86.

Edmonds, H.N., and Edmond, J.M., 1995. A three-component mixing model for ridge crest hydrothermal fluids. Earth Planet. Sci. Lett., 134:53-67.

Embley, R.W., Jonasson, I.R., Perfit, M.R., Franklin, J.M., Tivey, M.A., Malahoff, A., Smith, M.F., and Francis, T.J.G., 1988. Submersible investigation of an extinct hydrothermal system on the Galapagos Ridge: sulfide mounds, stockwork zone, and differentiated lavas. Can. Mineral., 26:517-539.

Fouquet, Y., von Stackelberg, U., Charlou, J.L., Donval, J.P., Foucher, J.P., Erzinger, J., Herzig, P., Mühe, R., Wiedicke, M., Soakai, S., and Whitechurch, H., 1991. Hydrothermal activity in the Lau back-arc basin: sulfides and water chemistry. Geology, 19:303-306.

Hannington, M.D., Jonasson, I.R., Herzig, P.M., and Petersen, S., 1995. Physical, chemical processes of seafloor mineralization at mid-ocean ridges. In Humphris, S.E., Zierenberg, R.A., Mullineaux, L.S., and Thomson, R.E. (Eds.), Seafloor Hydrothermal Systems: Physical, Chemical, Biological and Geological Interactions. Am. Geophys. Union Monogr., 91:115-157.

Hannington, M.D., Petersen, S., Jonasson, I.R., and Franklin, J.M., 1994. Hydrothermal activity and associated mineral deposits on the seafloor. Geol. Surv. Can., Open File Rep., 2915C.

Hannington, M.D., and Scott, S.D., 1988. Mineralogy and geochemistry of an hydrothermal silica-sulfide-sulfate spire in the caldera of Axial-Seamount, Juan de Fuca Ridge. Can. Mineral., 26:603-625.

Herzig, P.M., and Hannington, M.D., 1995. Polymetallic massive sulfides at the modern seafloor-a review. Ore Geol. Rev., 10:95-115.

Humphris, S.E., Herzig, P.M., Miller, D.J., Alt, J.C., Becker, K., Brown, D., Brügmann, G., Chiba, H., Fouquet, Y., Gemmell, J.B., Guerin, G., Hannington, M.D., Holm, N.G., Honnorez, J.J., Itturino, G.J., Knott, R., Ludwig, R., Nakamura, K., Petersen, S., Reysenbach, A.-L., Rona, P.A., Smith, S., Sturz, A.A., Tivey, M.K., and Zhao, X., 1995. The internal structure of an active sea-floor massive sulphide deposit. Nature, 377:713-716

Ito, E., and Anderson, A.T., Jr., 1983. Submarine metamorphism of gabbros from the Mid-Cayman Rise: petrographic and mineralogic constraints on hydrothermal processes at slow-spreading ridges. Contrib. Mineral. Petrol., 82:371-388.

Kelley, D.S., and Delaney, J.R., 1987. Two-phase separation and fracturing in mid-ocean ridge gabbros at temperatures greater than $700^{\circ} \mathrm{C}$. Earth Planet. Sci. Lett., 83:53-66.

Kelley, D.S., Gillis, K.M., and Thompson, G., 1993. Fluid evolution in submarine magma-hydrothermal systems at the Mid-Atlantic Ridge. J. Geophys. Res., 98:19579-19596.

Kelley, D.S., Robinson, P.T., and Malpas, J.G., 1992. Processes of brine generation and circulation in the oceanic crust: fluid inclusion evidence from the Troodos ophiolite, Cyprus. J. Geophys. Res., 97:9307-9322.

Kleinrock, M.C., and Humphris, S.E., 1996. Structural control on hydrothermalism at the TAG active mound, $26^{\circ} \mathrm{N}$. Nature, 382:149-153.

Kleinrock, M.C., Humphris, S.E., and the Deep-TAG Team, 1996. Detailed structure and morphology of the TAG active hydrothermal mound and its geotectonic environment. In Humphris, S.E., Herzig, P.M., Miller, D.J., et al., Proc. ODP, Init. Repts., 158: College Station, TX (Ocean Drilling Program), 15-21.

Lalou, C., Reyss, J.L., Brichet, E., Arnold, M., Thompson, G., Fouquet, Y., and Rona, P.A., 1993. New age data for Mid-Atlantic Ridge hydrother- mal sites: TAG and Snakepit geochronology revisited. J. Geophys. Res., 98:9705-9713.

Lalou, C., Reyss, J.-L., Brichet, E., Rona, P.A., and Thompson, G., 1995. Hydrothermal activity on a $10^{5}$-year scale at a slow-spreading ridge, TAG hydrothermal field, Mid-Atlantic Ridge $26^{\circ}$ N. J. Geophys. Res., 100:17855-17862.

Lalou, C., Thompson, G., Arnold, M., Brichet, E., Druffel, E., and Rona, P.A., 1990. Geochronology of TAG and Snakepit hydrothermal fields, Mid-Atlantic Ridge: witness to a long and complex hydrothermal history. Earth Planet. Sci. Lett., 97:113-128.

Le Bel, L., and Oudin, E., 1982. Fluid inclusion studies of deep-sea hydrothermal sulphide deposits on the East Pacific Rise near $21^{\circ} \mathrm{N}$. Chem. Geol., 37:129-136.

Leg 106 Scientific Party, 1986. Drilling the Snake Pit hydrothermal sulfide deposit on the Mid-Atlantic Ridge, lat. $23^{\circ} 22$ N. Geology, 14:10041007

Leitch, C.H.B., 1991. Preliminary studies of fluid inclusions in barite from the Middle Valley sulphide mounds, northern Juan de Fuca Ridge. Geol. Surv. Can. Pap., 91-1A:27-30.

Nehlig, P., 1991. Salinity of oceanic hydrothermal fluids: a fluid inclusion study. Earth Planet. Sci. Lett., 102:310-325.

Palmer, M.R., 1992. Controls over the chloride concentration of submarine hydrothermal vent fluids: evidence from $\mathrm{Sr} / \mathrm{Ca}$ and ${ }^{87} \mathrm{Sr} /{ }^{86} \mathrm{Sr}$ ratios. Earth Planet. Sci. Lett., 109:37-46.

Peter, J.M., Goodfellow, W.D., and Leybourne, M.I., 1994. Fluid inclusion petrography and microthermometry of the Middle Valley hydrothermal system, northern Juan de Fuca Ridge. In Mottl, M.J., Davis, E.E., Fisher, A.T., and Slack, J.F. (Eds.), Proc. ODP, Sci. Results, 139: College Station, TX (Ocean Drilling Program), 411-428.

Peter, J.M., and Scott, S.D., 1988. Mineralogy, composition, and fluid-inclusion microthermometry of seafloor hydrothermal deposits in the southern trough of Guaymas Basin, Gulf of California. Can. Mineral., 26:567587.

Roedder, E., 1984. Fluid inclusions. Rev. Mineral., Mineral. Soc. Am., 12.

Rona, P.A., Hannington, M.D., Raman, C.V., Thompson, G., Tivey, M.K., Humphris, S.E., Lalou, C., and Petersen, S., 1993. Active and relict seafloor hydrothermal mineralization at the TAG hydrothermal field, MidAtlantic Ridge. Econ. Geol., 88:1987-2013.

Rona, P.A., Klinkhammer, G., Nelson, T.A., Trefry, J.H., and Elderfield, H., 1986. Black smokers, massive sulfides and vent biota on the Mid-Atlantic Ridge. Nature, 321:33-37.

Rona, P.A., and Scott, S.D., 1993. A special issue on sea-floor hydrothermal mineralization: new perspectives. Econ. Geol., 88:1935-1975.

Saccocia, P.J., and Gillis, K.M., 1995. Hydrothermal upflow zones in the oceanic crust. Earth Planet. Sci. Lett., 136:1-16.

Seyfried, W.E., Jr., Berndt, M.E., and Janecky, D.R., 1986. Chloride depletions and enrichments in seafloor hydrothermal fluids: constraints from experimental basalt alteration studies. Geochim. Cosmochim. Acta, 50:469-475.

Shepherd, T.J., 1981. Temperature-programmable, heating-freezing stage for microthermometric analysis of fluid inclusions. Econ. Geol., 76:12441247.

Spooner, E.T.C., and Bray, C.J., 1977. Hydrothermal fluids of sea water salinity in ophiolitic sulphide ore deposits in Cyprus. Nature, 266:808812.

Thompson, G., Humphris, S.E., Schroeder, B., Sulanowska, M., and Rona, P.A., 1988. Active vents and massive sulfides at $26^{\circ} \mathrm{N}$ (TAG) and $23^{\circ} \mathrm{N}$ (Snakepit) on the Mid-Atlantic Ridge. Can. Mineral., 26:697-711.

Tivey, M.K., Humphris, S.E., Thompson, G., Hannington, M.D., and Rona, P.A., 1995. Deducing patterns of fluid flow and mixing within the TAG active hydrothermal mound using mineralogical and geochemical data. $J$. Geophys. Res., 100:12527-12555.

Vanko, D.A., 1986. High-chlorine amphiboles from oceanic rocks: product of highly saline hydrothermal fluids? Am. Mineral., 71:51-59.

, 1988. Temperature, pressure, and composition of hydrothermal fluids with their bearing on the magnitude of tectonic uplift at mid-ocean ridges, inferred from fluid inclusions in oceanic layer 3 rocks. J. Geophys. Res., 93:4595-4611.

Vanko, D.A., Griffith, J.D., and Erickson, C.L., 1992. Calcium-rich brines and other hydrothermal fluids in fluid inclusions from plutonic rocks, Oceanographer Transform, Mid-Atlantic Ridge. Geochim. Cosmochim. Acta, 56:35-47. 
Von Damm, K.L., 1990. Seafloor hydrothermal activity: black smoker chemistry and chimneys. Аnпи. Rev. Earth Planet. Sci., 18:173-204.

Von Damm, K.L., and Bischoff, J.L., 1987. Chemistry of hydrothermal solutions from the southern Juan de Fuca Ridge. J. Geophys. Res., 92:11,334-11,346.

Von Damm, K.L., Oosting, S.E., Kozlowski, R., Buttermore, L.G., Colodner, D.C., Edmonds, H.N., Edmond, J.M., and Grebmeier, J.M., 1995. Evolution of East Pacific Rise hydrothermal vent fluids following a volcanic eruption. Nature, 375:47-50.
Zhang, Y., and Frantz, J.D., 1987. Determination of the homogenization temperatures and densities of supercritical fluids in the system $\mathrm{NaCl}-\mathrm{KCl}-$ $\mathrm{CaCl}_{2}-\mathrm{H}_{2} \mathrm{O}$ using synthetic fluid inclusions. Chem. Geol., 64:335-350.

Date of initial receipt: 5 June 1996

Date of acceptance: 11 November 1996

Ms 158SR-210 

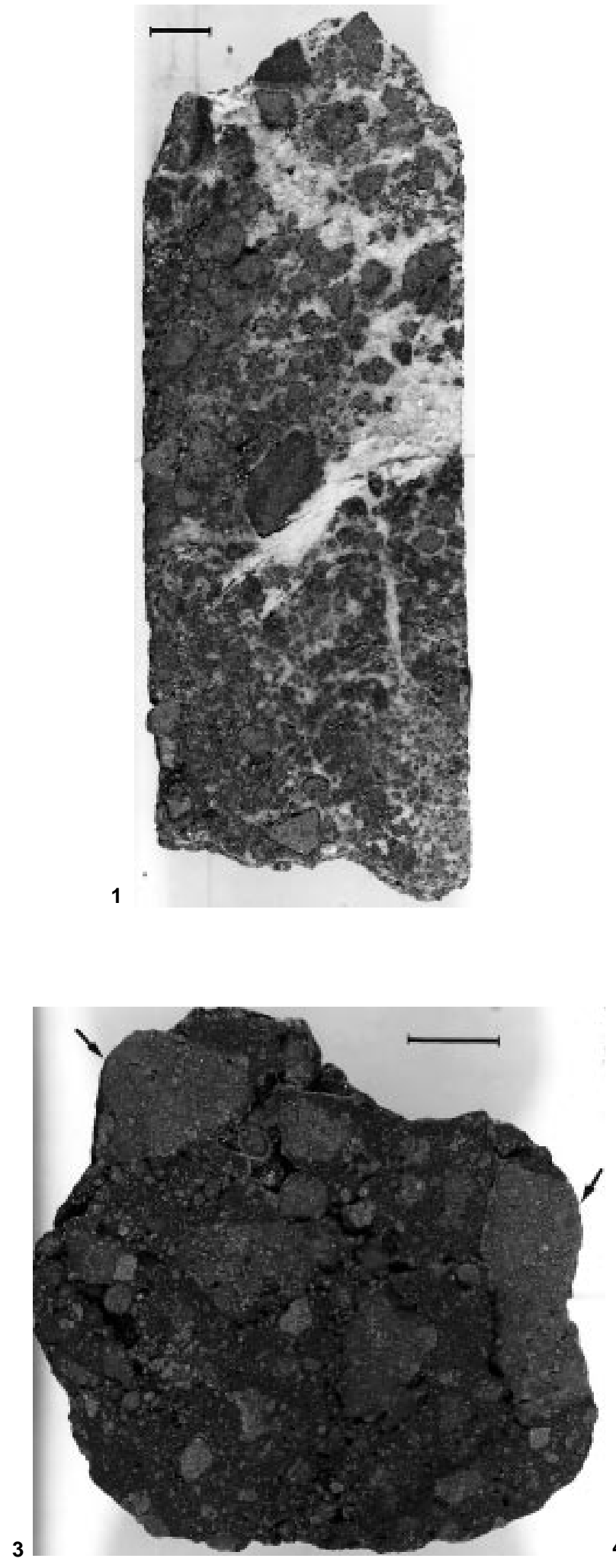

4

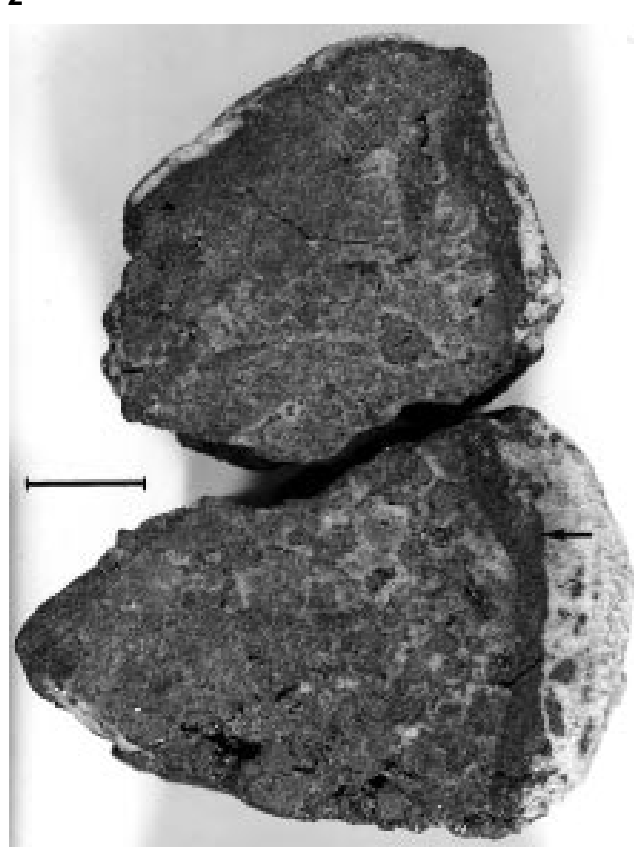

Plate 1. Photographs of representative samples from the interior of the TAG mound. 1. Nodular siliceous pyrite-anhydrite breccia from the anhydrite-zone showing rounded clasts of recrystallized pyrite and pyrite-silica intergrowth in a matrix of gray silica and white anhydrite. TAG-1 area; Sample 158-957C-7N-1 (Piece 8E), 118-133 cm. Scale bar is $1 \mathrm{~cm}$. 2. Nodular pyrite-silica breccia from the TAG-2 area characterized by subrounded light-gray quartz clasts in a matrix of dark quartz with abundant pyrite and vein-related, white anhydrite. Sample 158-957H-5N-1 (Piece 7), 65-77 cm. Scale bar is $1 \mathrm{~cm}$. 3. Pyrite-silica breccia with abundant subangular, gray quartz clasts (arrow) and recrystallized pyrite in a dark gray siliceous matrix with fine-grained disseminated pyrite. TAG-4 area; Sample 158-957M-2R-1 (Piece 7), 29-35 cm. Scale bar is $1 \mathrm{~cm}$. 4. Silicified wallrock breccia with pyrite aggregates in a gray siliceous matrix. The sample is rimmed by vein-related anhydrite (white) accompanied by a strong chalcopyritization (arrow). TAG-1 area, Sample 158-957E-4R-1 (Piece 1A, B), 0-7.5 cm. Scale bar is $1 \mathrm{~cm}$. 

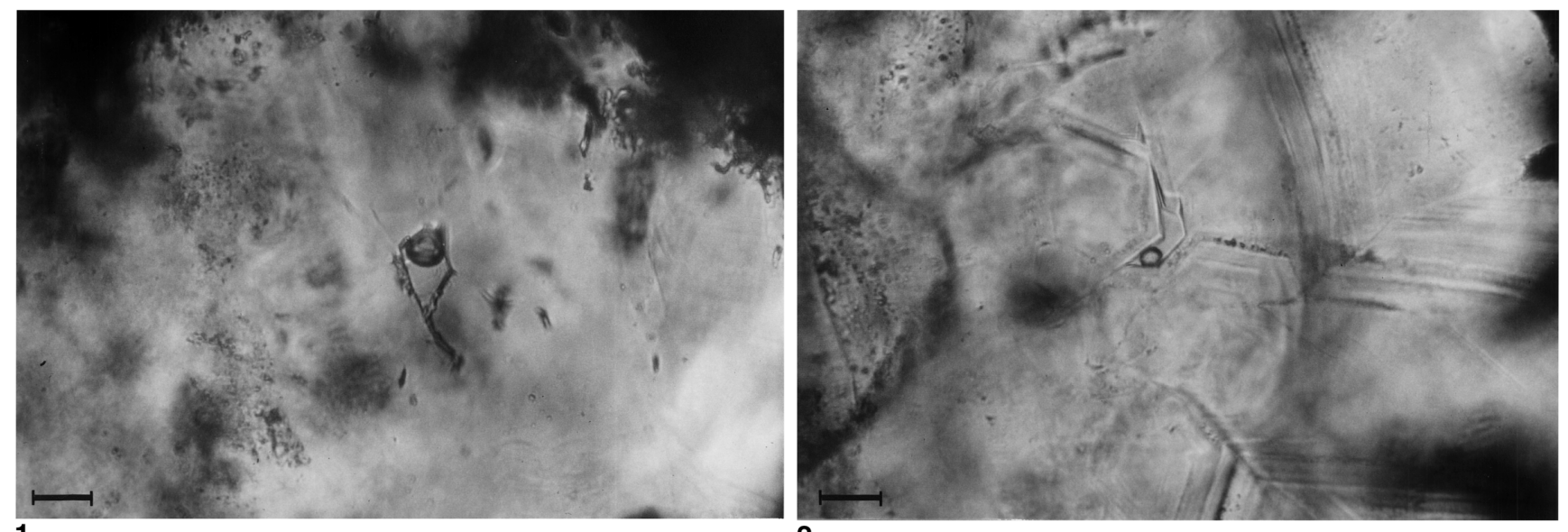

1
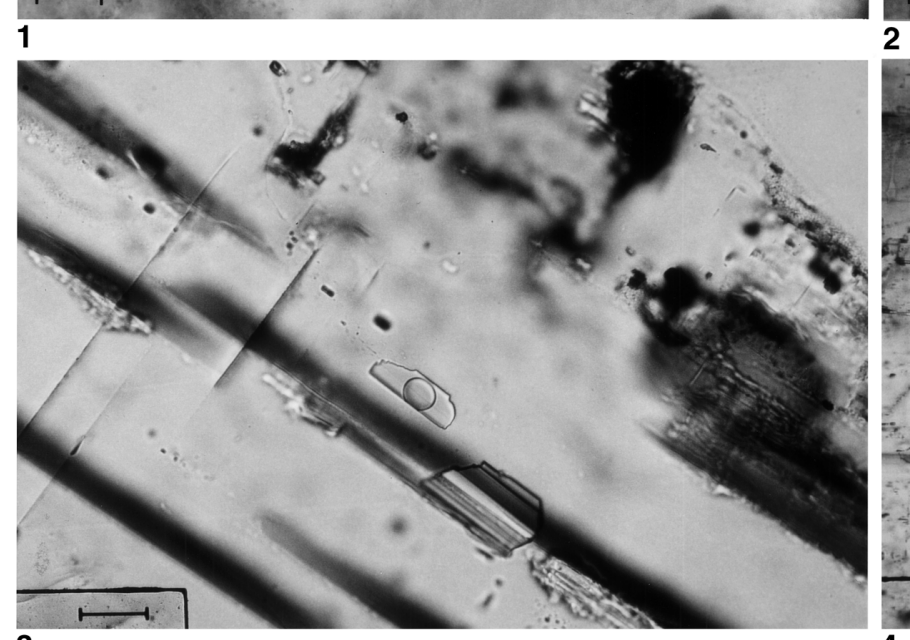

2

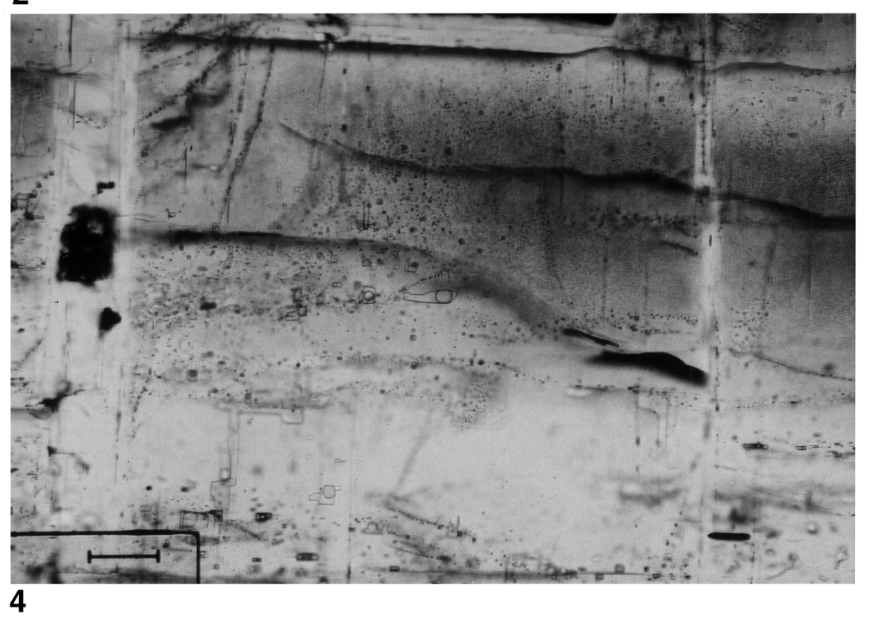

Plate 2. Fluid inclusions in quartz and anhydrite from the active TAG mound. 1. Large primary fluid inclusion in quartz from the Sample 158-957M-7R-1 (Piece 1), 0-3 cm. Scale bar is $8 \mu \mathrm{m}$. 2. Fluid inclusion at grain boundary between fine-grained zoned quartz crystals in pyrite-silica breccia. Sample 158-957M-7R-1 (Piece 1), 0-3 cm. Scale bar is $8 \mu \mathrm{m}$. 3. Angular fluid inclusion in anhydrite. Sample 158-957C-7N-2 (Piece 1K), 148-149 cm. Scale bar is $20 \mu \mathrm{m}$. 4. Abundant fluid inclusions and fluid inclusion trails in anhydrite. The anhydrite is broken and healed by transparent anhydrite. Sample 158-957C-7N-2 (Piece 1K), 148$149 \mathrm{~cm})$. Scale bar is $20 \mu \mathrm{m}$. 